\title{
Prinzipal Parlament. Die Bedeutung europäischer Parlamente als Wahlorgane
}

\author{
Ulrich Sieberer
}

Zusammenfassung: Parlamente sind in parlamentarischen Regierungssystemen zentrale Machtverteilungsorgane und als solche Prinzipale der Regierung und weiterer externer Amtsträger. Dieser Beitrag zeigt, dass parlamentarische Wahlbefugnisse im Rahmen eines delegationstheoretischen Modells erstens Delegationsverluste reduzieren und zweitens zu Abweichungen von der idealtypischen parlamentarischen Delegationskette führen können. Ihre Analyse erlaubt Rückschlüsse auf das tatsächliche Ausmaß der in bestehenden Modellen nur angenommenen Beschränkung der Regierung durch externe Amtsträger. Empirisch werden erstmals die Wahlbefugnisse von 25 europäischen Parlamenten bei der Besetzung von sieben Staatsämtern systematisch vergleichend untersucht. Die Analyse zeigt deutliche Unterschiede zwischen den untersuchten Parlamenten und identifiziert Wahlbefugnisse als eine auch empirisch distinkte Dimension parlamentarischer Machtbefugnisse.

Schlagwörter: Parlament $\cdot$ Regierung $\cdot$ Wahlfunktion $\cdot$ Delegationstheorie $\cdot$ Macht

Abstract: In parliamentary systems of government, parliaments can be conceptualized as central power-distributing institutions and as principals of the cabinet and other external officeholders. Relying on the principal-agent framework, this paper shows that electoral powers of parliaments can reduce agency loss and indicate a deviation from the ideal typical chain of delegation in parliamentary systems. Electoral powers of parliaments can be used to assess the degree to which cabinets are indeed constrained by external officeholders, whose constraining effect is often simply assumed. Empirically, the paper offers the first systematic comparative study of electoral powers of 25 European parliaments with regard to seven state offices. The analysis reveals major differences between parliaments and identifies electoral powers as an empirically distinct dimension of parliamentary power resources.

Keywords: Parliament $\cdot$ Government $\cdot$ Elective Function $\cdot$ Principal-agent-theory $\cdot$ Power

Ulrich Sieberer, M.A., (凸)

Universität Mannheim, Lehrstuhl für Politische Wissenschaft III, 68131 Mannheim,

E-Mail: sieberer@uni-mannheim.de 
The elective is now the most important function of the House of Commons. (...) It may be said that the House of Commons does not rule, it only elects the rulers. Walter Bagehot (1964 [1867]: 151, 157)

\section{Einleitung*}

Spätestens seit Lord Bryces (1921) berühmter These vom „Niedergang der Parlamente“ besteht in der Literatur weitgehende Einigkeit darüber, dass die zentralen inhaltlichen Politikentscheidungen in parlamentarischen Regierungssystemen in der Regel nicht im Parlament, sondern in der Regierung fallen (Gallagher et al. 2006: 57). Dieser Befund wird hauptsächlich auf die dominante Rolle von Parteien zurückgeführt, die die Präferenzen politischer Akteure über institutionelle Grenzen hinweg vereinheitlichen. Im Gegensatz zum klassischen Dualismus zwischen Regierung und Parlament führt dies zu einem „neuen Dualismus“, bei dem die entscheidenden politischen Konfliktlinien quer durch das Parlament - zwischen Regierungsfraktionen und der mit ihr verbundenen Regierung einerseits und Oppositionsfraktionen andererseits - verlaufen (Andeweg/ Nijzink 1995; von Beyme 1999; Döring 1994; Steffani 1997: 25-26, 148-151).

In der neueren Literatur werden aus dem neuen Dualismus unterschiedliche Schlüsse hinsichtlich der Notwendigkeit und Zielrichtung von Parlamentsforschung gezogen. Einige Autoren blenden die parlamentarische Arena völlig aus und konzentrieren sich nur auf die Interaktion von Regierungsparteien und möglichen außerparlamentarischen Vetospielern (z. B. Tsebelis 2002). Andere Autoren verweisen auf spezifische institutionelle Regelungen, über die parlamentarische Akteure weiterhin (wenn auch begrenzten) Einfluss auf Gesetzgebungsprozesse und -ergebnisse nehmen können (z.B. von Beyme 1997; Döring 1995a; Döring/Hallerberg 2004; Martin/Vanberg 2005). Eine dritte Gruppe richtet ihr Augenmerk verstärkt auf andere Facetten parlamentarischer Tätigkeit neben der Gesetzgebung, insbesondere auf die parlamentarische Kontrolle der Regierung (Helms 2005; Schnapp/Harfst 2005).

Im Sinne dieser dritten Gruppe nehme ich eine multifunktionale Perspektive ein und argumentiere, dass das Parlament in parlamentarischen Regierungssystemen auch als zentrales Machtverteilungsorgan konzeptualisiert werden sollte. Eine wichtige Aufgabe des Parlaments als in der Regel ${ }^{1}$ einzigem vom Volk gewählten und damit direkt legitimierten Organ besteht in der Auswahl weiterer Amtsträger, die ihrerseits inhaltliche Politikentscheidungen treffen. In delegationstheoretischer Terminologie ist das Parlament, genauer gesagt die Parlamentsmehrheit, also der Prinzipal der Regierung (Strøm 2000, 2003) und weiterer politisch relevanter Amtsträger.

* Für wertvolle Hinweise und Anregungen danke ich Thomas Gschwend, Matthias Lehnert, Eric Linhart, Gerhard Loewenberg, Bernhard Miller, Wolfgang C. Müller, Susumu Shikano, Thomas Zittel, den Teilnehmern des DVPW-Workshops „Parlamentarismus im politischen System der BRD“ in Bad Urach und eines Forschungskolloquiums am Mannheimer Zentrum für Europäische Sozialforschung (MZES) sowie zwei anonymen Gutachtern der PVS.

1 Ich behandle auch semipräsidentielle Systeme aufgrund der parlamentarischen Abberufbarkeit der Regierung als parlamentarisch (Müller et al. 2003). In diesen Systemen wird mit dem Staatspräsidenten ein weiteres Organ direkt vom Volk gewählt. 
Die Konzeptualisierung des Parlaments als Machtverteilungsorgan und Prinzipal anderer Amtsträger identifiziert parlamentarische Wahlbefugnisse als einen zentralen Gegenstand der Parlamentsforschung und als eine wichtige Machtressource parlamentarischer Akteure. Parlamentarische Wahlbefugnisse werden zwar in allen gängigen parlamentarischen Funktionskatalogen erwähnt (Bagehot 1964 [1867]2; Loewenberg/ Patterson 1979; Norton 1990; Patzelt 2003; Schüttemeyer 2003), wurden aber bisher weder theoretisch in neuere Konzepte zur Analyse parlamentarischer Demokratien integriert noch in ihrer empirischen Bedeutung systematisch vergleichend untersucht. Theoretische Argumente zur Bedeutung von Wahlen beschränken sich auf die Wahl von Parlamentariern und ansatzweise der Regierung (Fearon 1999; Strøm 2000, 2003). Empirisch untersucht wurden in Europa nur die Wahl der Regierung durch das Parlament (Bergman 1993) sowie die Wahl von Parlamentspräsidenten und anderen innerparlamentarischen Amtsträgern (Carroll et al. 2006; Jenny/Müller 1995). Nur für die USA wurde die Besetzung weiterer außerparlamentarischer Ämter durch das Parlament ausführlicher analysiert, da Verfassungsrichter und Amtsträger in verschiedenen Regulierungsbehörden nach ihrer Nominierung durch den Präsidenten die Zustimmung des Senats benötigen (z.B. Chang 2001; Epstein/Segal 2005; Krehbiel 2007; Moraski/ Shipan 1999; Nixon 2004).

Der vorliegende Beitrag setzt an diesen theoretischen und empirischen Forschungslücken an und analysiert die Rolle europäischer Parlamente als Wahlorgane. Dabei stehen drei Fragen im Mittelpunkt: (1) Regierungssysteme integrieren, und welche Folgen haben sie? (2) Wie stark sind europäische Parlamente als Wahlorgane? (3) Inwieweit bilden Wahlbefugnisse tatsächlich eine empirisch eigenständige Dimension parlamentarischer Machtbefugnisse?

Ich argumentiere erstens, dass parlamentarische Wahlbefugnisse schlüssig in ein delegationstheoretisches Modell parlamentarischer Regierungssysteme integriert werden können. Durch ihre Analyse kann dieses Modell hinsichtlich der Einbeziehung externer Organe und der Beurteilung, inwieweit man diese tatsächlich als Beschränkungen der Regierung ansehen kann, verbessert werden (Abschnitt 2). Zweitens stelle ich drei Indizes zur Messung der Stärke von Parlamenten als Wahlorganen vor (Abschnitt 3) und demonstriere, dass sich 25 europäische Parlamente in ihrer Bedeutung als Wahlorgane stark unterscheiden (Abschnitte 4 und 5). Drittens zeige ich, dass parlamentarische Wahlbefugnisse in Westeuropa eine auch empirisch eigenständige Dimension parlamentarischer Machtbefugnisse darstellen (Abschnitt 6). Abschließend diskutiere ich weitere Perspektiven für die Analyse parlamentarischer Wahlen sowie die Bedeutung meines Ansatzes über die Parlamentsforschung hinaus für durch Delegation gekennzeichnete politische Kontexte (Abschnitt 7).

2 Bagehots Beschreibung des britischen Unterhauses als Wahlorgan der Regierung ist allerdings unzutreffend, da die britische Regierung gerade nicht vom Parlament gewählt wird. Großbritannien ist damit ein Paradebeispiel für den ,negativen Parlamentarismus“ ohne explizite Regierungswahl (Bergman 1993). 


\section{Wahlen im Parlament als Abweichungen von der idealtypischen parlamentarischen Delegationskette}

Parlamentarische Regierungssysteme sind durch ein charakteristisches Spannungsverhältnis gekennzeichnet. Einerseits orientiert sich ihr institutioneller Rahmen weiterhin an klassischen Gewaltenteilungsvorstellungen und spricht ganz überwiegend von Rechten „des Parlaments“, oft im Gegensatz zu Rechten der Regierung. Andererseits ist die Akteurskonstellation geprägt vom neuen Dualismus zwischen Regierungs- und Oppositionsparteien, sodass sich die Präferenzen verschiedener Akteure innerhalb des Parlaments stark unterscheiden und dem Kollegialorgan Parlament kaum einheitliche Interessen zugeschrieben werden können. Angesichts dieses Spannungsverhältnises muss jede Untersuchung parlamentarischer Machtbefugnisse und ihrer Folgen in einen größeren Theorierahmen eingebettet sein, der die parlamentarische Ebene im Kontext des gesamten politischen Entscheidungsprozesses konzeptioniert. Dieser Rahmen muss sowohl mögliche Präferenzunterschiede zwischen Akteuren in unterschiedlichen institutionellen Arenen zulassen (d.h. die parlamentarische Arena darf nicht per Annahme für irrelevant erklärt werden) als auch die häufige Gleichrichtung von Akteurspräferenzen über Arenen hinweg integrieren können. Darüber hinaus sollte der Theorierahmen sowohl an die internationale Forschung anschlussfähig als auch in einer problemorientierten Perspektive über die Parlamentsforschung hinaus anwendbar sein (siehe z.B. Kaiser 2007; Patzelt 2004).

Diese Anforderungen legen die Verwendung der Delegationstheorie (principal-agenttheory) nahe, mit deren Hilfe unterschiedliche institutionelle Arenen und deren jeweilige Zusammenhänge in einem gemeinsamen und einfachen Analyserahmen untersucht werden können (Przeworski et al. 1999; Strøm et al. 2003a). Dieser Rahmen ist über die Parlamentsforschung hinaus in allen Kontexten anwendbar, in denen Entscheidungsbefugnisse delegiert werden. Er betont die Möglichkeit von Präferenzunterschieden auf unterschiedlichen Stufen des Delegationsprozesses und die daraus resultierende Gefahr von Delegationsverlusten. Ein eigenständiger Einfluss der parlamentarischen Arena ist damit nicht a priori ausgeschlossen. Gleichzeitig lassen sich Parteien als Mechanismen zur Vereinheitlichung von Akteurspräferenzen über Arenen hinweg systematisch in die Theorie einbeziehen (Müller 2000). Schließlich unterstreicht die Delegationstheorie die große Bedeutung der Auswahl von Amtsträgern (,Agenten“) und die Vorteile, die einem Wahlorgan („Prinzipal“) aus der Bestellung erwachsen (Fearon 1999).

Delegationstheoretische Modelle repräsentativ-demokratischer Systeme gehen von der Feststellung aus, dass politische Entscheidungen in modernen Demokratien in aller Regel nicht direkt vom Prinzipal Volk, sondern von verschiedenen direkt oder indirekt gewählten Agenten getroffen werden. Diese Delegation ist aus Sicht des Prinzipals rational: aufgrund eigener Kapazitätsbegrenzungen, besonderer Kompetenzen der Agenten sowie der Möglichkeit, durch Delegation Probleme kollektiven Entscheidens zu umgehen (Strøm 2003: 56-59). ${ }^{3}$ Andererseits birgt sie die Gefahr von Delegationsverlusten, da Agenten die ihnen notwendigerweise überlassenen Handlungsspielräume gegen die

3 Der letzte Punkt gilt nur eingeschränkt, wenn der Agent selbst ein Kollegialorgan wie ein Parlament ist, in dem Probleme kollektiven Entscheidens auftreten können. 
Interessen des Prinzipals nutzen können (Lupia 2003). Grundsätzlich lassen sich zwei Strategien unterscheiden, mit denen Prinzipale Delegationsverluste zu minimieren suchen: erstens die Bestellung von Agenten, deren Präferenzen mit denen des Prinzipals übereinstimmen und die daher aufgrund ihrer eigenen Präferenzen im Sinne des Prinzipals handeln, sowie zweitens die Verknüpfung von illoyalem Agentenverhalten mit verschiedenen Kosten, die abweichendes Verhalten für den Agenten selbst bei andersgearteten persönlichen Präferenzen unattraktiv machen. Insbesondere bei der ersten Strategie spielt die Wahl von Amtsträgern eine zentrale Rolle, da sie die Auswahl von Agenten mit gleichlaufenden Präferenzen ermöglicht (,selecting good types“, Fearon 1999). Daneben kann die Wahl auch als Ex-post-Mechanismus zur Sanktionierung illoyaler Agenten dienen, wenn diese eine Wiederwahl anstreben (,sanctioning poor performance", Fearon 1999). ${ }^{4}$ Die Wahl von Amtsträgern ist also eine wichtige Machtressource des Prinzipals zur Minimierung von Delegationsverlusten.

Aus delegationstheoretischer Sicht lassen sich parlamentarische Regierungssysteme idealtypisch als einfache und indirekte Delegationsketten verstehen, in denen politische Entscheidungsgewalt in vier Schritten vom Volk als ultimativem Prinzipal an (1) das Parlament (gemeint ist immer nur diejenige Kammer, von deren Vertrauen die Regierung abhängt), (2) den Regierungschef und das Kabinett, (3) die zuständigen Fachminister und schließlich (4) die Ministerialbürokratie delegiert wird (Strøm 2000, 2003). In dieser Kette delegiert jeder Prinzipal nur an einen (oder mehrere, nicht miteinander konkurrierende) Agenten; jeder Agent ist im Gegenzug jeweils nur einem Prinzipal verantwortlich. Das Parlament fungiert demnach als Agent der Wählerschaft, und die Parlamentsmehrheit ist ihrerseits der Prinzipal des Regierungschefs und seiner Regierung. ${ }^{5}$ Das Parlament gestaltet inhaltliche Politik demnach nicht selbst, sondern bestimmt und kontrolliert den damit beauftragten Agenten Regierung. Das Parlament ist also in dieser Konzeptualisierung in erster Linie Machtverteilungsorgan, nicht autonomer Gesetzgeber.

Aufgrund der langen, indirekten Delegationskette und des weitgehenden Fehlens nachträglicher Kontrollmechanismen legen parlamentarische Demokratien besonderes Gewicht auf die Auswahl von Agenten mit übereinstimmenden Präferenzen. Dabei spielen politische Parteien eine zentrale Rolle, da sie die Präferenzen von Akteuren auf unterschiedlichen Stufen des Delegationsprozesses gleichrichten (Müller 2000). Idealtypisch ist die parlamentarische Demokratie also gleichzeitig ,party government" (Cox 1987a).

4 Noch direkter ist die Ex-post-Kontrolle, wenn Agenten durch den Prinzipal abgewählt und wiedergewählt werden können. Die Möglichkeiten zur Abberufbarkeit und Wiederwahl werden hier aus Platzgründen ausgeblendet. Empirisch führt diese Einschränkung nicht zu Verzerrungen, da nur in neun der hier untersuchten 165 Fälle das Parlament einen Amtsträger wählt, der weder abberufbar ist noch wiedergewählt werden kann.

5 Auch wenn die Regierung gerade in Koalitionskabinetten streng genommen kein unitarischer Akteur ist, verfolgt sie doch ein gemeinsames Programm, das häufig in Koalitionsverträgen festgelegt und/oder in Koalitionsausschüssen ausgehandelt wird. Die Beziehung zwischen Koalitionspartnern und die dort angewendeten Kontrollmechanismen werden in diesem Beitrag ausgeblendet. 
In der idealtypischen parlamentarischen Demokratie findet eine Wahl im Parlament nur zur Bestellung des Agenten Regierung statt; die Regierung ihrerseits delegiert spezifische Entscheidungsbefugnisse an eigene Agenten, insbesondere die Ministerialbürokratie. Die Wahl der Regierung gewährleistet eine weitgehende Präferenzübereinstimmung zwischen Prinzipal und Agent. In vielen parlamentarischen Demokratien (unter anderem Großbritannien, das häufig als beste empirische Annäherung an den Idealtyp parlamentarischer Demokratien verstanden wird) findet eine solche formale Regierungswahl indes nicht statt. Stattdessen wird die parlamentarische Akzeptanz einer vom Staatsoberhaupt ernannten Regierung vorausgesetzt, solange ihr nicht explizit das Misstrauen ausgesprochen wird. Diese Abweichung wird aus zwei Gründen oft als relativ unproblematisch eingeschätzt. Erstens existiert in all diesen Systemen per definitionem das Recht des Parlaments, die Regierung abzuberufen. Diese Abberufbarkeit verhindert die Bildung von Regierungen gegen den Willen einer parlamentarischen Mehrheit. Zweitens führt die zentrale Rolle politischer Parteien zu informellen Selektionsprozessen innerhalb der parlamentarischen Mehrheit, die auch ohne formale Wahl massive Delegationsverluste bei der Regierungsbildung verhindern. Allerdings erleichtert das Fehlen einer Regierungswahl oder eine Wahl, bei der keine absolute Mehrheit der Parlamentarier erforderlich ist, die Bildung von Minderheitsregierungen (Bergman 1993), die ihrerseits eine Abweichung von der idealtypischen Situation parlamentarischer Mehrheitsregierungen darstellen. Insofern ist es sinnvoll, die tatsächlichen institutionellen Regelungen der Regierungswahl zu betrachten.

Nicht nur bei der Regierungswahl weichen parlamentarische Demokratien empirisch von der idealtypischen Delegationskette ab. Konzeptionell nennt Strøm zwei weitere wichtige Abweichungen (Strøm 2003: 73-77; Strøm et al. 2003b): Erstens kann die Stärke von Parteien und damit deren Fähigkeit zur Gleichrichtung von Präferenzen zwischen Parlamentsmehrheit und Regierung variieren. Zweitens kann die Handlungsfreiheit der Regierung durch verschiedene institutionelle Regelungen eingeschränkt sein. Als zwei Arten solcher institutional constraints können die Aufteilung von Zuständigkeiten (partitions, z. B. föderale Strukturen, Korporatismus oder konkordanzdemokratische Arrangements) und institutionelle Kontrollen (checks, z. B. Verfassungsgerichte, Vetorechte des Staatspräsidenten oder abrogative Referenden) unterschieden werden (Strøm 2003: 76-77).

In Strøms Modell stehen diese institutionellen Schranken weitgehend unverbunden neben der Delegationskette. Damit bleibt zunächst unklar, inwieweit von ihnen tatsächlich eine Beschränkung der Regierung zu erwarten ist. Aus neoinstitutionalistischer Sicht setzt eine solche Beschränkung zweierlei voraus: Externe Amtsträger müssen erstens über ausreichende institutionelle Machtbefugnisse verfügen und zweitens aufgrund ihrer Präferenzen Anreize haben, diese zur Beschränkung der Regierung einzusetzen. Die institutionellen Machtbefugnisse werden in der vergleichenden Literatur häufig betrachtet (z.B. zu Staatspräsidenten Bergman et al. 2003; Siaroff 2003; zu Verfassungsgerichten Ishiyama Smithey/Ishiyama 2002; Hönnige 2007; zu Ombudsleuten Miller 2004). Zu den Präferenzen dieser Amtsträger hingegen existiert wenig Forschung, obwohl von einer effektiven Beschränkung der Regierung nur ausgegangen werden kann, wenn diese Präferenzen von denen der Regierung abweichen. 
Eine Analyse der zusätzlich einbezogenen Präferenzen erfordert zunächst eine Differenzierung der institutionellen Schranken dahingehend, ob durch sie Akteure der bestehenden Delegationskette zusätzliche Einflussmöglichkeiten bekommen (z. B. die gesamte Wählerschaft bei Referenden oder ein Teil der Wählerschaft bei föderalen Arrangements) oder ob neue Akteure mit institutioneller Macht ausgestattet werden (beispielsweise Staatspräsidenten oder Verfassungsrichter). ${ }^{6}$ Wenn neben den Akteuren der idealtypischen parlamentarischen Delegationskette weitere externe Amtsträger in den politischen Prozess einbezogen werden, können durch sie neue Präferenzen in den Prozess eingeführt werden, die tatsächlich zu einer Beschränkung der Regierung führen. $^{7}$

Systematische Erwartungen hinsichtlich der Präferenzen dieser externen Amtsträger lassen sich über den Modus ihrer Bestellung bilden. Mit Ausnahme der Direktwahl von Staatspräsidenten in semipräsidentiellen Systemen (Neto/Strøm 2006) wird diese Bestellung allerdings nicht thematisiert. Die Analyse von Wahlen in Parlamenten erlaubt Rückschlüsse auf die Präferenzen externer Amtsträger. Werden externe Amtsträger vom Parlament gewählt, sind deren Präferenzen weitgehend an die Präferenzen der sie wählenden parlamentarischen Akteure zurückgebunden. Die institutionelle Ausgestaltung des Wahlverfahrens erlaubt Aussagen über die Wahrscheinlichkeit, mit der Akteure, deren Präferenzen nicht mit denen der Regierung übereinstimmen, relevanten Einfluss auf den Wahlausgang haben. ${ }^{8}$

Im folgenden Abschnitt diskutiere ich, welche institutionellen Variablen des Wahlverfahrens für den Einfluss parlamentarischer Akteure auf den Wahlausgang und für die Wahrscheinlichkeit, dass die Handlungsfreiheit der Regierung durch die Wahl von Amtsträgern mit abweichenden Präferenzen tatsächlich eingeschränkt wird, prägend sind.

\section{Institutionelle Wahlmodelle und die Beschränkung der Regierung}

Unter parlamentarischer Wahl verstehe ich im Weiteren jedes Verfahren, bei dem ein außerparlamentarisches Amt nur mit expliziter Zustimmung der notwendigen parlamentarischen Mehrheit ausgeübt werden kann. Bei bikameralen Parlamenten wird nur die-

6 Diese Unterscheidung entspricht (mit Ausnahme der hier nicht behandelten direktdemokratischen Instrumente) der Differenzierung von ,internal“ und „external constraints" in Strøm et al. (2003b).

7 Werden institutionelle Schranken durch Akteure der bestehenden Delegationskette besetzt, sind deren Präferenzen bereits in das Modell integriert. Bei territorialer (Föderalismus) oder funktionaler (Korporatismus) Aufteilung des kollektiven Prinzipals Volk können aufgrund der unterschiedlichen Aggregationsprozesse Kollektivpräferenzen zum Tragen kommen, die ebenfalls zu einer zusätzlichen Beschränkung der Regierung führen. Dieser Aspekt bleibt in diesem Beitrag unberücksichtigt.

8 Institutionelle Machtbefugnisse externer Amtsträger werden in diesem Beitrag weitgehend ausgeblendet, stellen aber eine theoretisch und empirisch unabhängige zweite Dimension bei der Einschätzung der tatsächlichen Beschränkung der Regierung durch externe Amtsträger dar (Sieberer 2008). 
jenige Kammer betrachtet, der die Regierung verantwortlich ist. ${ }^{9}$ Diese Definition umfasst auch Situationen, in denen ein anderweitig nominierter Kandidat die Zustimmung des Parlaments benötigt. Auch wenn dieses Verfahren häufig nicht als „Wahl“, sondern als „parlamentarische Bestätigung“ bezeichnet wird, stellt es einen Mechanismus dar, über den die Präferenzen von Parlamentariern direkt relevant für die Besetzung werden. Auch verpflichtende Vertrauensabstimmungen über eine Regierung unmittelbar nach deren Amtsantritt sind Wahlen im Sinne meiner Definition.

Wie muss nun ein Wahlverfahren im Parlament institutionell ausgestaltet sein, damit es zu Beschränkungen der Regierung führt? Angesichts der oben getroffenen Unterscheidung eines alt-dualistischen Institutionenrahmens und einer neu-dualistischen Akteurskonstellation müssen institutionelle Beschränkungen der Regierung in einem Doppelschritt analysiert werden. Erstens ist im Sinne des alten Dualismus zu fragen, wie direkt die Präferenzen parlamentarischer Akteure auf das Wahlergebnis durchschlagen. Dies hängt institutionell von den Variablen (1) institutionell zulässige Kandidatenzahl und (2) nominierungsberechtigte Akteure ab. Zweitens muss im Sinne des neuen Dualismus untersucht werden, mit welcher Wahrscheinlichkeit aufgrund institutioneller Regelungen Akteure mit von der Regierung abweichenden Präferenzen in eine Wahlkoalition einbezogen werden müssen. Diese hängt ab von den Variablen (3) Mehrheitserfordernis und (4) Abstimmungsmodus. Auf der Grundlage dieser Variablen entwickle ich drei Indizes zur Messung der institutionellen Beschränkung der Regierung durch parlamentarische Wahlen.

Zuvor sind allerdings zwei Klarstellungen notwendig: Erstens schließt die hier unternommene institutionelle Betrachtung nicht aus, dass institutionelle Regeln in der Praxis von informellen Faktoren überlagert werden können. Dennoch bilden formale Regeln den Rahmen, innerhalb dessen sich informelle Praktiken ausbilden und stabilisieren können. So ist beispielsweise der informelle Lagerproporz bei der Besetzung von Richterposten am Bundesverfassungsgericht nur vor dem Hintergrund der für die Wahl notwendigen Zweidrittelmehrheit verständlich (Schulze-Fielitz 1984). In vielen Fällen werden die angesichts formaler Regeln erwarteten Unterschiede wegen informeller Praktiken nicht beobachtbar sein, insbesondere wenn sich Regierung und Regierungsfraktionen in ihren Präferenzen völlig einig sind. In den politisch wie politikwissenschaftlich interessanten Konstellationen mit Präferenzunterschieden hingegen kommt den im Weiteren analysierten formalen Befugnissen eine wichtige Rolle zu.

Zweitens ist mit der Nutzung von Indizes ein grundsätzliches Problem verbunden. Indizes sollen komplexe Konzepte erfassen, indem sie mehrere relevante und interagierende Variablen in einer Maßzahl zusammenfassen (Miller 2007). Diese Zusammenfassung erfordert bei der Indexkonstruktion Annahmen zur Art der Verknüpfung und zur Gewichtung von Variablen. Die im Weiteren verwendeten Setzungen werden erstens theoretisch begründet, zweitens wird empirisch gezeigt, dass plausible alternative Setzungen zu substanziell ähnlichen Ergebnissen führen. Die Ergebnisse des Beitrags sind also robust gegenüber verschiedenen Entscheidungen bei der Indexkonstruktion. Angesichts dieser Robustheit werden die Indizes möglichst einfach und für den Leser gut nachvollziehbar gehalten.

9 Zum Sonderfall Italien siehe Fußnote 27. 
Welche institutionellen Wahlmodelle lassen sich nun identifizieren? Auf der Basis der Kandidatenzahl werden zunächst zwei Grundmodelle unterschieden. Kann nur ein Kandidat zur Wahl stehen, spreche ich vom „Bestätigungsmodell“ (B), bei mehreren möglichen Kandidaten vom „kompetitiven Wahlmodell“ (KW). ${ }^{10}$ Der wichtigste Unterschied zwischen diesen beiden Modellen liegt in der unterschiedlichen Relevanz der Nominierung. Im Bestätigungsmodell hat der Nominierungsberechtigte den Vorteil des Agendasetzers, d.h. er kann einen für sich günstigen Kandidaten durchsetzen, selbst wenn dessen Position deutlich vom Idealpunkt des Parlaments abweicht. Die einzige Bedingung ist, dass der vorgeschlagene Kandidat von der notwendigen Mehrheit der Rückfalloption, d.h. in der Regel dem (kommissarischen) Verbleib des Amtsinhabers im Amt, vorgezogen wird. Mithilfe formaler Modellierung lässt sich zeigen, dass je nach Präferenzkonstellation der Idealpunkt des entscheidenden Parlamentsakteurs (je nach Mehrheitserfordernis des Parlamentsmedians oder des qualifizierten Mehrheitspivots, vgl. Krehbiel 2006) mehr oder weniger einflussreich für die Präferenzen des letztlich gewählten Agenten sein kann. Es gibt sogar Konstellationen, in denen von parlamentarischen Akteuren trotz abweichender Präferenzen keinerlei Beschränkung des Nominierungsorgans ausgeht (Romer/Rosenthal 1978; Moraski/Shipan 1999). Bei mehreren Kandidaten hingegen ist der Vorteil des Agendasetzers geringer. Wenn Kandidaten von mehreren konkurrierenden und strategisch handelnden Akteuren nominiert werden, sollten nach der Logik des Medianwählertheorems nur Kandidaten am Idealpunkt des Parlaments gewählt werden, selbst wenn der Medianabgeordnete selbst nicht nominierungsberechtigt ist (Hinich/Munger 1997: 21-49). ${ }^{11}$ Ist hingegen nur ein Akteur nominierungsberechtigt, kann dieser auch im kompetitiven Wahlmodell einen gewissen Agendasetzervorteil für sich nutzen, indem er mehrere Kandidaten nominiert, die sich nur geringfügig unterscheiden. Die Wahlmöglichkeiten des Parlaments sind also höher als im Bestätigungsmodell, eine perfekte Umsetzung der Präferenzen des entscheidenden Parlamentsakteurs ist allerdings nicht garantiert.

Innerhalb der beiden Grundmodelle unterscheide ich je drei Untertypen, je nachdem, von wem Kandidaten nominiert werden können. Dieses Recht kann (1) parlamentarischen Akteuren selbst (einzelnen Abgeordneten, einzelnen Fraktionen oder bestimmten innerparlamentarischen Amtsträgern; auch politische Parteien als Organisationen fallen in diese Gruppe), (2) Regierungsakteuren (dem Regierungschef, einem Fachminister oder dem Kabinett als Kollegialorgan) oder (3) dritten Organen (in der Regel dem Staatsoberhaupt oder dem zu besetzenden Organ selbst) zustehen. Für parlamentarische Akteure ist es natürlich am günstigsten, wenn sie Kandidaten selbst nominieren können. Die Regierung hingegen wird in ihrer Handlungsfreiheit am wenigsten eingeschränkt, wenn sie durch eigene Nominierungen zumindest den Agendasetzervorteil nutzen kann. Eine Nominierung durch ein außerhalb der Delegationskette stehendes drittes Organ

10 Empirisch wird auch in einer Wahl, in der die Aufstellung mehrerer Kandidaten möglich wäre, teils nur ein Kandidat nominiert. Eine solche Wahl fällt dennoch unter die Logik des kompetitiven Wahlmodells.

11 Dies gilt für absolute und (mit Einschränkungen, Cox 1987b) einfache Mehrheitswahl. Für qualifizierte Mehrheitserfordernisse sagt das kompetitive Wahlmodell keine Gleichgewichtslösung voraus. 
liegt in Bezug auf die Beschränkung der Regierung durch das Parlament zwischen den beiden Extremen.

Insgesamt lassen sich sechs Wahlmodelle unterscheiden, unter denen die Präferenzen parlamentarischer Akteure mehr oder weniger starken Einfluss auf das Ergebnis der Wahl haben (Tabelle 1). Für die Reihung dieser Modelle gehe ich davon aus, dass das Nominierungsrecht größeren Einfluss auf die Umsetzung der Präferenzen parlamentarischer Akteure hat als die Kandidatenzahl, da der Agendasetzervorteil des nominierenden Akteurs auch in zwei der drei Untertypen des kompetitiven Wahlmodells erhalten bleibt. Weder die Regierung noch ein externes drittes Organ können von parlamentarischen Akteuren gezwungen werden, mehrere signifikant unterschiedliche Kandidaten zu nominieren. Am stärksten ist der Einfluss parlamentarischer Akteure auf die Präferenzen der gewählten Amtsträger demnach im kompetitiven Wahlmodell mit parlamentarischer Nominierung, gefolgt vom Bestätigungsmodell mit diesem Nominierungsverfahren. Es folgen die beiden Modelle mit Nominierung durch ein externes drittes Organ sowie die Modelle mit Regierungsnominierung. Diese Entscheidung zum Vorrang einer der beiden Dimensionen ist für die empirische Analyse unkritisch, da der Index auf der Grundlage der alternativen Entscheidung zu sehr ähnlichen Ergebnissen führt. ${ }^{12}$ Aus der Reihung der sechs institutionellen Wahlmodelle ergibt sich direkt ein erster Index „Parlamentarische Wahlfreiheit". Dieser wird zwischen 0 und 1 skaliert, nimmt den Wert 0 an, wenn das entsprechende Amt ganz ohne Beteiligung des Parlaments besetzt wird und erhält den Wert 1 für das aus Sicht des Parlaments günstigste Wahlmodell KW1. Daneben wird angenommen, dass die Abstände zwischen den einzelnen Modellen gleich groß sind. ${ }^{13}$ Damit ergeben sich für die sechs unterschiedenen Wahlmodelle die in Tabelle 1 in Klammern angegebenen Indexwerte.

12 Für die in diesem Aufsatz untersuchten Wahlen korrelieren die Indizes auf der Grundlage der beiden Entscheidungen mit $\mathrm{r}=0,87$. Dieser Korrelation liegen nur die 86 Fälle mit parlamentarischer Wahl zugrunde, da Fälle ohne Wahl notwendigerweise auf beiden Indizes den Wert 0 erhalten. Unter Einbeziehung dieser Fälle ist der Zusammenhang noch stärker mit $r=0,96$ $(\mathrm{N}=165)$. Beide Zusammenhänge sind auf dem 1-Prozent-Niveau signifikant. Auch wenn es sich bei den verwendeten Daten um keine Stichprobe handelt, werden im Weiteren Signifikanztests durchgeführt. Der Grund liegt in möglichen Messfehlern. Es ist unklar, wie genau die verwendeten Indikatoren die zu messenden theoretischen Konzepte erfassen. Gerade angesichts der unten diskutierten Möglichkeiten verschiedener theoretisch plausibler und empirisch hoch, aber nicht perfekt korrelierter Operationalisierungen kann angenommen werden, dass die verwendeten Messwerte das Konzept nicht perfekt widerspiegeln. Gleichzeitig ist eine spezifische Richtung möglicher Abweichungen nicht ersichtlich, sodass die Messfehler als normalverteilt angenommen werden können. Unter diesen Voraussetzungen ist auch in einer Vollerhebung die Verwendung von Signifikanztests bei Korrelationskoeffizienten sinnvoll (zur Diskussion vgl. Behnke 2005; Broscheid/Gschwend 2005).

13 Diese Setzung kann sicherlich diskutiert werden. So könnte man beispielsweise annehmen, dass die Distanz zwischen den beiden Modellen mit gleichem Nominierungsrecht (z. B. KW1 und B1) geringer ist als diejenige zwischen zwei aufeinanderfolgenden Modellen mit unterschiedlichem Nominierungsrecht (z. B. B1 und KW2). Letztlich spiegelt der Abstand zwischen den Werten eine Annahme über die Wichtigkeit der beiden Dimensionen Nominierungsrecht und Kandidatenzahl wider. Die Annahme gleicher Abstände gewichtet beide Dimensionen gleich, da theoretisch über die im Text bereits diskutierte Reihung der Wahlmodelle hinaus keine klaren Indizien für den Vorrang einer Dimension vorliegen. In einem solchen Fall stel- 
Tabelle 1: Institutionelle Wahlmodelle und Werte des Index „Parlamentarische Wahlfreiheit“

\begin{tabular}{l|l|cccc}
\hline \multicolumn{4}{c}{} & \multicolumn{3}{c}{ Nominierungsberechtigtes Organ } \\
\multicolumn{3}{l|}{} & \multicolumn{1}{c}{ Parlament } & Drittes Organ & Regierung \\
\hline \multirow{3}{*}{$\begin{array}{l}\text { Institutionell zulässige } \\
\text { Kandidatenzahl }\end{array}$} & \multirow{3}{*}{ Einer } & $\mathrm{B} 1$ & $\mathrm{~B} 2$ & $\mathrm{~B} 3$ \\
& \multirow{2}{*}{ Mehrere } & $(0,83)$ & $(0,5)$ & $(0,17)$ \\
& & $\mathrm{KW} 1$ & $\mathrm{KW} 2$ & $\mathrm{KW} 3$ \\
& & $(1)$ & $(0,67)$ & $(0,33)$ \\
\hline
\end{tabular}

Anmerkungen: $\mathrm{B}=$ Bestätigungsmodell; $\mathrm{KW}=$ kompetitives Wahlmodell; (Indexwert Parlamentarische Wahlfreiheit; keine Beteiligung des Parlaments an Besetzung eines Amts $=0$ )

Aus der Perspektive des neuen Dualismus ist für die tatsächliche Beschränkung der Regierung nicht nur entscheidend, dass die Präferenzen parlamentarischer Akteure den Wahlausgang direkt beeinflussen, sondern auch wessen Präferenzen innerhalb des Parlaments ausschlaggebend sind. Die Regierung wird durch parlamentarische Wahlbefugnisse nur dann eingeschränkt, wenn für die Wahl eines Amtsträgers die Zustimmung von parlamentarischen Akteuren mit abweichenden Präferenzen notwendig ist.

Zwei Gruppen solcher Akteure sind hierbei relevant: Oppositionsfraktionen und Minderheiten innerhalb der Regierungsfraktionen, die in der Regierung selbst nicht vertreten sind. Beiden Gruppen können plausibel von der Regierung abweichende Präferenzen unterstellt werden, wobei das Einbeziehen von Oppositionsfraktionen die Regierung zu größeren Zugeständnissen zwingen sollte als die Rücksichtnahme auf innerfraktionelle Gruppen mit abweichenden Präferenzen. ${ }^{14}$

Ob Oppositionsfraktionen in eine Wahlkoalition einbezogen werden müssen, hängt von der erforderlichen Mehrheit als institutioneller Variable sowie von der Sitzverteilung im Parlament als situativer Variable ab. Ich betrachte hier nur das Mehrheitserfordernis als die institutionell vermittelte Wahrscheinlichkeit, dass die Zustimmung von Oppositionsakteuren für eine Wahl im Parlament notwendig ist. Zu unterscheiden sind einfache Mehrheit, absolute Mehrheit der Mitglieder des Parlaments und qualifizierte Mehrheiten, von denen die Dreifünftel- und die Zweidrittelmehrheit empirisch am häufigsten vorkommen.

Die einfache Mehrheitsregel gilt in den meisten Parlamenten als grundlegende Entscheidungsregel und schränkt die Regierung daher bei Wahlen kaum ein. Für Minderheitsregierungen ist diese Mehrheitsregel von Vorteil, da sie unter Umständen von einer Spaltung der Opposition profitieren können. Die absolute Mehrheitsregel erzwingt die

len ungewichtete Indizes und äquivalent dazu die Annahme gleicher metrischer Distanzen in meinen Augen die überzeugendste Rückfalloption dar, da sie erstens für den Leser leicht nachvollziehbar sind und zweitens keine ad hoc eingeführten Zusatzinformationen beinhalten.

14 Daneben sind Situationen denkbar, in denen Koalitionsparteien unterschiedliche Präferenzen in Bezug auf ein zu besetzendes Amt haben. Ich gehe davon aus, dass solche Konflikte zwischen den jeweiligen Führungen im Kabinett oder in einem Koalitionsausschuss gelöst werden, die diese Lösung dann in ihren jeweiligen Fraktionen durchsetzen müssen. Für diese Annahme spricht der empirische Befund, dass konkurrierende Nominierungen von Koalitionsparteien sehr selten sind. 
Bildung „echter“ Mehrheitskoalitionen, was für Mehrheitsregierungen normalerweise unproblematisch ist. Bei Minderheitsregierungen ist hingegen die explizite Zustimmung von Oppositionsfraktionen notwendig, da ein Regierungskandidat nicht mehr von Enthaltungen oder mehreren, sich gegenseitig schwächenden Kandidaten aus dem Oppositionslager profitieren kann. Qualifizierte Mehrheitserfordernisse schließlich erzwingen für gewöhnlich die Zustimmung von Oppositionsparteien, es sei denn, die Regierung verfügt ausnahmsweise über die notwendige qualifizierte Mehrheit.

Aus den oben diskutierten sechs Wahlmodellen und den drei Mehrheitserfordernissen bilde ich einen zweiten Index der Beschränkung der Regierung aufgrund parlamentarischer Wahlbefugnisse. Diese Beschränkung ist umso stärker, je freier das Parlament einerseits in der Umsetzung seiner Präferenzen ist und je eher andererseits die Zustimmung von Oppositionsfraktionen notwendig ist. Diese beiden Aspekte verstärken sich gegenseitig, d.h. die Beschränkung der Regierung steigt überproportional, wenn die Präferenzen parlamentarischer Akteure entscheidend sind und Oppositionsfraktionen zustimmen müssen. Diese gegenseitige Verstärkung wird durch eine multiplikative Verknüpfung der Elemente des Index erreicht (Wehner 2006). Der Index „Regierungsbeschränkung I" errechnet sich daher als das Produkt des Index „Parlamentarische Wahlfreiheit" und der Variablen Mehrheitserfordernis. Diese Variable nimmt die Werte 1 für einfache Mehrheit, 1,5 für absolute Mehrheit und 2 für qualifizierte Mehrheit an. Durch diese Werte ist gewährleistet, dass die beiden Elemente des neuen Index in etwa gleich stark auf die Reihung der Indexwerte verschiedener Wahlmodelle wirken und keines der Elemente einseitig dominiert. ${ }^{15}$ Empirisch ist der Index robust gegenüber anderen Wertzuweisungen für die Variable Mehrheitserfordernis. ${ }^{16}$

Beschränkungen der Regierung können schließlich nicht nur dadurch entstehen, dass Oppositionsfraktionen einbezogen werden, sondern können auch aus der erzwungenen Rücksichtnahme auf Minderheiten innerhalb der eigenen Fraktionen resultieren. Trotz der hohen beobachteten Geschlossenheit von Fraktionen in parlamentarischen Abstimmungen (Sieberer 2006) dürften aus drei Gründen Präferenzunterschiede zwischen Regierungsmitgliedern und Teilen der Regierungsfraktionen existieren: Erstens muss sich die Präferenzheterogenität von Fraktionen im Kabinett keineswegs vollständig wider-

15 Dies zeigt sich an den jeweils höchsten und niedrigsten Werten des Index Regierungsbeschränkung I: Den höchsten Wert hat das Modell KW1 mit qualifizierter Mehrheit, gefolgt von B1 mit qualifizierter Mehrheit, KW1 mit absoluter Mehrheit, KW2 mit qualifizierter Mehrheit und B1 mit absoluter Mehrheit. Unter den Modellen mit den höchsten Werten variieren also alle drei in den Index eingehenden Variablen (Kandidatenzahl, Nominierungsorgan, Mehrheitserfordernis). Dasselbe gilt für die niedrigsten Indexwerte. Diese fallen auf die Modelle B3 mit einfacher Mehrheit, B3 mit absoluter Mehrheit, KW3 mit einfacher Mehrheit, B3 mit qualifizierter Mehrheit, B2 mit einfacher Mehrheit sowie KW3 mit absoluter Mehrheit. Der niedrigste Wert 0 wird natürlich wiederum in Fällen vergeben, in denen keine Wahl im Parlament stattfindet.

16 Verwendet man die Faktoren 1 (einfache Mehrheit), 2 (absolute Mehrheit) und 3 (qualifizierte Mehrheit), korrelieren dieser und der von mir verwendete Index mit $r=0,96(p<0,01)$. Verwendet man für beide Vercodungsentscheidungen (Reihung der Dimensionen im Index Parlamentarische Wahlfreiheit und Mehrheitserfordernis) die alternativen Vercodungen, beträgt die Korrelation $r=0,91(p<0,01)$. Beide Zusammenhänge liegen noch höher, wenn Fälle mit Indexwert 0 einbezogen werden. 
spiegeln, insbesondere wenn kleinen Regierungsparteien nur wenige Kabinettsposten zur Verfügung stehen. Zweitens entwickeln Regierungsmitglieder aufgrund ihrer Regierungstätigkeit teilweise Präferenzen, die von denen ihrer Fraktionskollegen abweichen, sei es dadurch, dass sie in eine Ministeriumskultur einbezogen werden (,going native"), oder durch die Konfrontation mit externen Anforderungen, Finanzzwängen oder internationalen Einbindungen (Andeweg 2000). Drittens sind Fraktionshinterbänkler elektoral verwundbarer als die meist gut abgesicherten Kabinettsmitglieder und haben daher ein essenzielles Interesse an einer gewissen, wenn auch in der Regel nur begrenzt öffentlich sichtbaren Kontrolle der Regierung.

$\mathrm{Ob}$ sich diese abweichenden Präferenzen individueller Parlamentarier in parlamentarischem Abstimmungsverhalten allgemein und bei Wahlen im Parlament im Speziellen manifestieren, hängt davon ab, inwieweit die Fraktionsführungen in der Lage sind, individuelles Abstimmungsverhalten zu beobachten und abweichendes Verhalten zu sanktionieren (Saalfeld 1995). Institutionell ist hierfür die Unterscheidung zwischen geheimen Wahlen einerseits und namentlichen oder halboffenen Wahlen (beispielsweise durch Handzeichen) andererseits entscheidend. Bei geheimen Wahlen sind die Überwachungsmöglichkeiten der Fraktionsführung beschränkt, entsprechend unsicherer ist der Wahlausgang und entsprechend stärker müssen die Präferenzen innerfraktioneller Minderheiten im Vorfeld der Wahl berücksichtigt werden.

Diese zusätzliche Beschränkung durch innerfraktionelle Minderheiten wird im Index „Regierungsbeschränkung II" integriert. Eine multiplikative Verknüpfung würde die Bedeutung des Abstimmungsmodus allerdings überschätzen. Stattdessen wähle ich eine additive Verknüpfung und erhöhe den jeweiligen Indexwert von Regierungsbeschränkung I um 0,33, wenn eine Wahl geheim stattfindet. ${ }^{17}$ Durch die Addition eines festen Betrags ist die Bedeutung der Variable Abstimmungsverhalten für den Gesamtindex am höchsten, wenn die Regierung aufgrund des Wahlmodells und der erforderlichen Mehrheit wenig beschränkt ist. Dies entspricht der Beobachtung, dass in solchen Fällen die wichtigste Aufgabe der Regierung darin liegt, die Zustimmung in den eigenen Reihen sicherzustellen. Ist hingegen die Zustimmung von Oppositionsfraktionen erforderlich, werden innerfraktionelle Einwände eher mit dem Hinweis auf Kompromisszwänge mit der Opposition zurückgewiesen.

Bevor die drei Indizes zur empirischen Analyse der Wahlbefugnisse europäischer Parlamente genutzt werden, diskutiere ich im nächsten Abschnitt die Auswahl der untersuchten Länder und zu besetzenden Ämter, stelle die verwendeten Datenquellen vor und erkläre einige generelle Vercodungs- und Aggregationsentscheidungen.

17 Der addierte Betrag von 0,33 entspricht einem Drittel der insgesamt möglichen Varianz des Index Parlamentarische Wahlfreiheit und erfasst so die theoretisch geringere Bedeutung der Variable Abstimmungsmodus. Diese Setzung ist wiederum empirisch nicht kritisch. Indizes aufgrund alternativer Vercodungen mit den Beträgen 0,25 und 0,5 korrelieren mit dem von mir verwendeten Index mit $r=0,99(p<0,01)$. 


\section{Fallauswahl und Daten}

Bei der Fallauswahl sind zwei Entscheidungen begründungsbedürftig: Welche Parlamente werden analysiert, und die Besetzung welcher Ämter wird untersucht? Mein theoretisches Argument zu Wahlen im Parlament als möglichen Abweichungen von der idealtypischen Delegationskette bezieht sich auf parlamentarische Regierungssysteme. Diese müssen ein Mindestmaß an Stabilität des demokratischen Systems aufweisen, damit eine prägende Wirkung von Institutionen auf Akteursverhalten angenommen werden kann. Innerhalb der theoretisch relevanten Gruppe stabiler parlamentarischer Demokratien analysiere ich die parlamentarischen Systeme der 25 EU-Mitgliedsstaaten zum Stichtag 1. Januar 2006 (d.h. alle damaligen EU-Staaten mit Ausnahme des präsidentiellen Systems Zyperns) sowie Norwegen $(\mathrm{N}=25)$. Diese Länderauswahl garantiert eine weitgehende Anschlussfähigkeit an die bestehende Forschung zu europäischen Demokratien.

Die zu analysierenden Ämter außerhalb des Parlaments wurden nach folgenden Kriterien ausgewählt: Ein Amt muss dauerhaft politisch und theoretisch relevant sein, muss über die untersuchten Länder hinweg äquivalente Aufgaben erfüllen. Die Ergebnisse sollten an andere Arbeiten der vergleichenden Institutionenforschung anschließen können. Aufgrund dieser Kriterien untersuche ich parlamentarische Wahlbefugnisse bei der Besetzung von sieben Ämtern, die sich wiederum in drei Gruppen unterteilen lassen: (1) Regierungschef und Minister als zentrale Politikentscheider in parlamentarischen Demokratien und idealtypisch einzige Agenten des Parlaments, (2) Staatspräsidenten, Verfassungsrichter und Zentralbankpräsidenten als „Entscheider“, die zumindest in einzelnen Politikfeldern oder bei einzelnen prozeduralen Fragen direkten Einfluss auf politisch relevante Entscheidungen nehmen können, sowie (3) Rechnungshofspräsidenten und Ombudsleute als „Kontrolleure“ ohne eigene Entscheidungskompetenz.

Die politische und theoretische Relevanz von Regierungschef und Ministern steht außer Frage. Staatspräsidenten, Verfassungsrichter und Zentralbankpräsidenten sind sicherlich relevante politische Akteure, auch wenn ihr Einfluss auf Politikinhalte zwischen Ländern variiert. Viele Staatspräsidenten verfügen über eigene Befugnisse in der Gesetzgebung, der Regierungsbildung, der Parlamentsauflösung oder der Ernennung weiterer Amtsträger. Auch Staatspräsidenten ohne weitreichende formale Befugnisse können als „mächtige Spieler“ im Sinne Strøms (2003b: 77) bezeichnet werden, die beispielsweise durch öffentliche Äußerungen die Auszahlungen von Vetospielern und damit indirekt deren Verhalten beeinflussen können. Verfassungsgerichte können Gesetze für verfassungswidrig erklären und besitzen damit oft Vetomacht. Zentralbankpräsidenten haben wichtige Entscheidungsbefugnisse in der Geldpolitik, sowohl auf nationaler Ebene als auch auf europäischer Ebene als Mitglieder des Rats der Europäischen Zentralbank. Die Aufgabe von Rechnungshofspräsidenten und Ombudsleuten ${ }^{18}$ hingegen besteht hauptsächlich in der Überwachung der Regierung und der ihr nachgeordneten Verwaltung; häufig werden diese Organe explizit als Hilfsorgane des Parlaments

18 Betrachtet wurden nur Ombudsleute mit umfassender Zuständigkeit, nicht hingegen Institutionen, die nur für einen bestimmten Bereich, beispielsweise das Militär, Kinder oder Minderheiten, zuständig sind. 
bezeichnet. Die von ihnen generierten Informationen können von interessierten Akteuren, insbesondere Oppositionsfraktionen, im politischen und elektoralen Wettbewerb genutzt werden. Dies verringert Informationsasymmetrien zwischen Regierungs- und Parlamentsakteuren und reduziert so die Gefahr abweichenden Verhaltens der amtierenden Regierung (moral hazard). Beide Institutionen haben in der Literatur bisher wenig Beachtung gefunden (National Audit Office 2005; Miller 2004).

Die untersuchten Ämter unterscheiden sich offensichtlich in ihrer politischen Bedeutung. Sie werden hier dennoch gemeinsam untersucht, um eine umfassende Einschätzung der Rolle von Parlamenten als Wahlorganen zu erhalten. Diese Rolle sollte nicht einseitig auf die Regierungswahl beschränkt werden. Gleichzeitig wird im Weiteren gezeigt, dass unterschiedliche Gewichtungen nach der Bedeutung der einzelnen Ämter kaum zu starken Veränderungen führen. Man kann also sinnvoll von „der“ institutionellen Macht eines Parlaments als Wahlorgan sprechen.

Insgesamt liegen der empirischen Untersuchung 165 Besetzungsverfahren zu den sieben Ämtern in 25 Staaten zugrunde, wobei hier insbesondere die 86 Fälle interessieren, in denen das Parlament an der Besetzung im Sinne meiner Wahldefinition beteiligt ist. ${ }^{19}$ Die institutionellen Daten wurden aus den relevanten Rechtsnormen (Verfassungen, parlamentarischen Geschäftsordnungen und einschlägigen Spezialgesetzen, wie Verfassungsgerichtsgesetzen, Zentralbankgesetzen etc.), den Internetseiten der Parlamente und der $\mathrm{zu}$ besetzenden Organe, der Sekundärliteratur (insbesondere Ismayr 1999, 2004; Strøm et al. 2003a) sowie individuellen schriftlichen Anfragen an alle untersuchten Parlamente erhoben.

Drei generelle Vercodungsentscheidungen sind erklärungsbedürftig: Erstens wurden mehrere institutionell unterschiedliche Wahlgänge zunächst einzeln vercodet. Für die folgenden Analysen wird fast immer der entscheidende letzte Wahlgang verwendet, der in der Regel der für die Regierung günstigste Wahlgang ist. Selbst wenn diese Wahlgänge empirisch selten zum Einsatz kommen, ist zu vermuten, dass Akteure diese Möglichkeit antizipieren und in ihre Entscheidungen in früheren Wahlgängen einbeziehen. Davon ausgenommen sind nur Fälle, in denen parlamentarische Akteure in einem früheren Wahlgang bei gleichem Mehrheitserfordernis eine stärkere Position aufgrund des Nominierungsrechts innehaben und daher versuchen sollten, diese Stärke bereits vor dem letzen Wahlgang auszunutzen. ${ }^{20}$ Werden zweitens Amtsträger von gemischten Versammlungen aus Parlamentariern und anderen Akteuren gewählt (beispielsweise bei der Wahl des deutschen Bundespräsidenten durch die Bundesversammlung), wird der Indexwert mit dem Anteil der Parlamentarier in der Versammlung gewichtet, um deren Einfluss innerhalb der gemischten Versammlung zu erfassen. Wählt drittens das Parla-

19 Die Abweichung von der rechnerischen Fallzahl von 175 entsteht, da in acht Ländern kein Verfassungsgericht und in zwei Ländern kein Ombudsman existiert.

20 Bei der Wahl des polnischen Premierministers wurde deshalb der zweite Wahlgang mit parlamentarischem Nominierungsrecht anstelle des dritten mit einem Nominierungsmonopol des Staatspräsidenten verwendet. Bei der Wahl des deutschen Bundeskanzlers wurde der zweite Wahlgang mit absoluter Mehrheit zugrunde gelegt, da eine Wahl mit relativer Mehrheit im dritten Wahlgang dem Bundespräsidenten die freie Entscheidung überlässt, den gewählten Minderheitenkanzler zu ernennen oder den Bundestag aufzulösen (Art. 63 GG). Aufgrund dieser Unsicherheit dürften parlamentarische Akteure diese Option nicht verfolgen. 
ment nur einen Teil der Amtsträger in einem Kollegialorgan (was vor allem bei Verfassungsgerichten häufig vorkommt), wird der Indexwert mit dem Anteil der vom Parlament gewählten Amtsträger gewichtet, da Parlamentarier nur die Präferenzen derjenigen Amtsträger beeinflussen können, an deren Auswahl sie beteiligt sind. ${ }^{21}$

Die oben vorgestellten Indizes werden zunächst für jedes zu besetzende Amt einzeln gemessen und anschließend für jedes Land addiert und durch die Zahl der dort existierenden Ämter geteilt. ${ }^{22}$ Bei dieser Aggregation stellen sich zwei Gewichtungsfragen: Erstens sind manche der untersuchten Ämter politisch offensichtlich relevanter als andere. Insbesondere die Regierung hat eine Sonderstellung inne, aber auch Verfassungsgerichten wird größere politische Bedeutung beigemessen als beispielsweise Ombudsleuten. Zweitens variieren die institutionellen Kompetenzen der zu besetzenden Ämter teilweise stark zwischen Ländern. Aufgrund dieser Überlegung wurde eine Reihe unterschiedlicher Gewichtungen verwendet, um zu überprüfen, wie stark die empirischen Ergebnisse von Gewichtungsentscheidungen abhängen.

Zunächst wurden die drei Ämtergruppen (Regierung - Entscheider - Kontrolleure) im Verhältnis 3:2:1 gewichtet, was die unterschiedliche Bedeutung der einzelnen Ämtergruppen erfasst, ohne Aussagen darüber zu machen, um wie viel bedeutsamer diese jeweils sind. Zweitens wurde aufgrund der besonders hervorgehobenen Bedeutung der Regierung eine Gewichtung von 4:2:1 verwendet. Drittens wurde ein ungewichteter Index ohne Einbeziehung der Wahl von Regierungschef und Ministern berechnet.

Die drei Indizes erweisen sich beim Vergleich der aufgrund unterschiedlicher Gewichtungsentscheidungen entstandenen Werte als sehr robust. Der ungewichtete Index Parlamentarische Wahlfreiheit über alle Ämter korreliert mit den beiden gewichteten Indizes mit $r=0,93$ (Gewichtung 3:2:1) bzw. $r=0,91$ (4:2:1) und mit dem ungewichteten Index ohne Regierungsämter mit $r=0,92$. Für die Indizes Regierungsbeschrän-

21 Implizit geht diese Gewichtung davon aus, dass jeder Amtsträger innerhalb des zu besetzenden Kollegialorgans gleichen Einfluss auf dessen Entscheidungen hat. Die Auswahl von beispielsweise einem Drittel der italienischen Verfassungsrichter durch das Parlament führt demnach dazu, dass dieses über die Auswahl entsprechender Richter die Entscheidungen des Verfassungsgerichts zu einem Drittel kontrolliert. Bei Mehrheitsentscheidungen innerhalb des Gerichts und einer räumlichen Entscheidungslogik ist diese Annahme problematisch. Streng genommen liegt die Entscheidungsmacht beim Gerichtsmedian. Wer diesen allerdings bestellt, ließe sich nur situativ auf der Grundlage der Präferenzen der relevanten Parlamentsmehrheit und der weiteren Bestellungsorgane (im Beispielsfall des Staatspräsidenten und der obersten Gerichte) beurteilen. Dies ist für größere Fallzahlen unmöglich. Die Annahme gleichen Einflusses aller Amtsträger innerhalb eines Kollegialorgans stellt eine notwendige und meines Erachtens insgesamt plausible Annäherung an die sicherlich komplexere Entscheidungslogik dieser Organe dar.

22 Die Division durch die Zahl der pro Land existierenden Ämter ist einer Division durch die Zahl der analysierten Ämter vorzuziehen, da ansonsten die Nichtexistenz eines Amtes denselben Effekt hätte wie die Besetzung dieses Amtes durch die Regierung selbst (Indexwert 0). Die Regierung kann aber einen von ihr selbst ausgewählten externen Amtsträger zur Legitimierung ihrer Politik nutzen, beispielsweise indem verfassungsrechtliche Bedenken der Opposition durch ein „,neutrales“, aber von der Regierung besetztes Verfassungsgericht autoritativ zurückgewiesen werden oder ein Ombudsman der Regierung effiziente Verwaltungstätigkeit attestiert. 
kung I und II sind die Zusammenhänge noch etwas stärker, die Korrelationskoeffizienten liegen zwischen 0,92 und $0,96(\mathrm{p}<0,01, \mathrm{~N}=25)$.

Die unterschiedliche Machtfülle desselben Amts zwischen Ländern ist schwerer zu integrieren, da dazu vergleichende Daten über die Befugnisse der jeweiligen Amtsträger erforderlich sind. Solche Daten stehen für Rechnungshofspräsidenten und Ombudsleute nicht für alle untersuchten Länder zur Verfügung. Bei Zentralbankpräsidenten dürfte die Varianz aufgrund der für alle EU-Staaten (nicht nur die Staaten der Eurozone) verbindlichen Vorgaben der EZB (Art. 14 Satzung der EZB) gering sein. Auch die Bedeutung von Regierungen ist wohl über die Länder hinweg vergleichbar; institutionelle Varianz besteht eher hinsichtlich der Machtverteilung innerhalb der Regierung und der Rolle des Regierungschefs (Bergman et al. 2003: 177-196), die für die Relevanz der Wahl der Regierung als Kollegialorgan durch das Parlament allerdings nicht direkt bedeutsam ist.

Die Macht von Staatspräsidenten und Verfassungsgerichten hingegen wird in der Literatur als zwischen Ländern stark unterschiedlich beschrieben. Diese Varianz wurde durch Siaroffs Index zur institutionellen Macht von Staatspräsidenten (Siaroff 2003) sowie den ,judicial power score“ erfasst, der institutionelle Machtbefugnisse von Verfassungsgerichten misst (Ishiyama Smithey/Ishiyama 2002; die dort nicht behandelten westeuropäischen Verfassungsgerichte wurden auf Grundlage der jeweiligen Verfassungen, der Internetseiten der Verfassungsgerichte sowie Informationen aus Hönnige 2007 vercodet). Diese Machtindizes wurden so standardisiert, dass sie zwischen 0,5 und 1,5 variieren und einen Mittelwert von 1 haben. Anschließend wurden die Indexwerte der entsprechenden Wahlverfahren mit diesem Faktor gewichtet. Die Wahl eines institutionell starken Amtsträgers weist einem Parlament damit einen höheren Wert zu als die Wahl eines schwachen Amtsträgers.

Empirisch hat das Einbeziehen der länderspezifisch unterschiedlichen Bedeutung von Staatspräsidenten und Verfassungsgerichten nahezu keinen Effekt. Die Korrelationen zwischen den Indizes mit und ohne diese Gewichtung betragen je nach Index und sonstiger Gewichtung zwischen 0,98 und $0,99 .{ }^{23}$ Da gerade Staatspräsidenten und Verfassungsgerichte als Ämter mit starker Varianz im Hinblick auf ihre institutionelle Macht gelten, ist zu erwarten, dass auch dann keine größeren Auswirkungen auf die empirischen Ergebnisse zu erwarten sein dürften, wenn (leider nicht vorhandene) Daten zur Macht von Rechnungshofspräsidenten und Ombudsleuten einbezogen würden.

Die beständig sehr hohen Korrelationen der Indizes nach unterschiedlichen Gewichtungsverfahren (und, wie in Abschnitt 3 demonstriert, unter unterschiedlichen Vercodungsentscheidungen) zeigen, dass die Indexwerte nicht auf die in der Indexkonstruktion und -aggregation unumgänglichen Setzungen zurückzuführen sind. Ebenso machen sie deutlich, dass die Werte einzelner Länder nicht vorrangig auf der starken Rolle bei der Bestellung nur eines Amtsträgers beruhen, da sich ansonsten stärkere Unterschiede zwischen den gewichteten und ungewichteten Indizes zeigen müssten. ${ }^{24}$ Diese Befunde

$23 p<0,01$. Die hohe Korrelation ist auch darauf zurückzuführen, dass die nach Siaroff (2003) stärksten Präsidenten in Frankreich und Litauen direkt vom Volk gewählt werden. Die tatsächlich von Parlamenten gewählten Staatspräsidenten sind in ihrer institutionellen Machtfülle mit Werten zwischen 0 und 2 auf dem Neunpunkteindex von Siaroff relativ homogen.

24 Dafür spricht auch, dass meine Indizes hoch mit der Zahl von Ämtern korreliert, an deren Besetzung ein Parlament beteiligt ist. 
verstärken das Vertrauen in die Robustheit der Indizes und machen die Wahl der Gewichtung weniger kritisch, da die Ergebnisse sich kaum unterscheiden. Ich verwende im Folgenden die für den Leser leicht nachvollziehbaren ungewichteten Indizes über alle Ämter ohne Einbeziehung länderspezifischer Unterschiede.

\section{Die institutionelle Macht europäischer Parlamente als Wahlorgane}

Betrachtet man zunächst die Verteilung der drei Indizes anhand einfacher deskriptiver Statistiken, zeigen alle drei Indizes empirisch breite Varianz (Tabelle 2). Der theoretische Minimalwert von 0 wird erreicht, da die französische Nationalversammlung bei keinem der untersuchten Ämter über Wahlbefugnisse verfügt. Die theoretisch denkbaren Maximalwerte kommen empirisch nicht vor; allerdings wird für alle Indizes in etwa die Hälfte des möglichen Wertebereichs ausgeschöpft. Die beobachteten Werte sind statistisch annähernd normalverteilt. Die Indizes sind also geeignet, die untersuchten Parlamente im Hinblick auf ihre Bedeutung als Wahlorgane zu differenzieren.

Tabelle 2: Deskriptive Statistiken zu parlamentarischen Wahlbefugnissen in 25 europäischen Demokratien

\begin{tabular}{lccc}
\hline & $\begin{array}{c}\text { Parlamentarische } \\
\text { Wahlfreiheit }\end{array}$ & $\begin{array}{c}\text { Regierungs- } \\
\text { beschränkung I }\end{array}$ & $\begin{array}{c}\text { Regierungs- } \\
\text { beschränkung II }\end{array}$ \\
\hline Mittelwert & 0,37 & 0,46 & 0,56 \\
Standardabweichung & 0,15 & 0,20 & 0,26 \\
Beobachtete Wertespanne & $0-0,64$ & $0-0,87$ & $0-1,06$ \\
(Theoretische Wertespanne) & $(0-1,00)$ & $(0-2,00)$ & $(0-2,33)$ \\
Median & 0,38 & 0,46 & 0,59 \\
Unteres Quartil (25\%) & 0,32 & 0,33 & 0,42 \\
Oberes Quartil (75\%) & 0,43 & 0,59 & 0,69 \\
\hline
\end{tabular}

Quelle: eigene Daten.

Die drei Indizes erfassen unterschiedliche Konzeptualisierungen parlamentarischer Macht. Der Index Parlamentarische Wahlfreiheit beruht auf der institutionellen Unbeschränktheit des Parlaments in der Umsetzung seiner Präferenzen, die beiden anderen Indizes erfassen die institutionelle Beschränkung der Regierung durch parlamentarische Wahlbefugnisse, entweder nur aufgrund der notwendigen Zustimmung von Oppositionsparteien oder zusätzlich durch die notwendige Rücksichtnahme auf Minderheiten innerhalb der Regierungsfraktionen. Trotz dieser Unterschiede zeigt die empirische Analyse hoch signifikante und stark positive Zusammenhänge aller drei Indizes. ${ }^{25}$ Die Indizes Regierungsbeschränkung I und II korrelieren sehr stark mit $\mathbf{r}=0,95$, und auch der nach der Logik des alten Dualismus konstruierte Index Parlamentarische Wahlfreiheit weist mit $r=0,83$ bzw. $r=0,81$ starke Zusammenhänge mit den beiden neu-dua-

25 Ein Teil dieser Zusammenhänge ist durch die Indexbildung bedingt, da die Indizes Regierungsbeschränkung I bzw. II auf der Grundlage der Indizes Parlamentarische Wahlfreiheit bzw. Regierungsbeschränkung I berechnet werden. 
listischen Indizes Regierungsbeschränkung I und II auf. ${ }^{26}$ Eine Analyse der Streudiagramme zeigt weiterhin, dass diese Zusammenhänge nicht auf Ausreißer zurückzuführen sind.

Angesichts der hohen Zusammenhänge beschränke ich mich im Weiteren auf den Index Regierungsbeschränkung II, der die umfassendste Konzeptualisierung der Beschränkung der Regierung aufgrund parlamentarischer Wahlbefugnisse enthält. Die Werte der untersuchten Parlamente sind in Abbildung 1 dargestellt.

Abbildung 1: Indexwerte Regierungsbeschränkung II nach Ländern

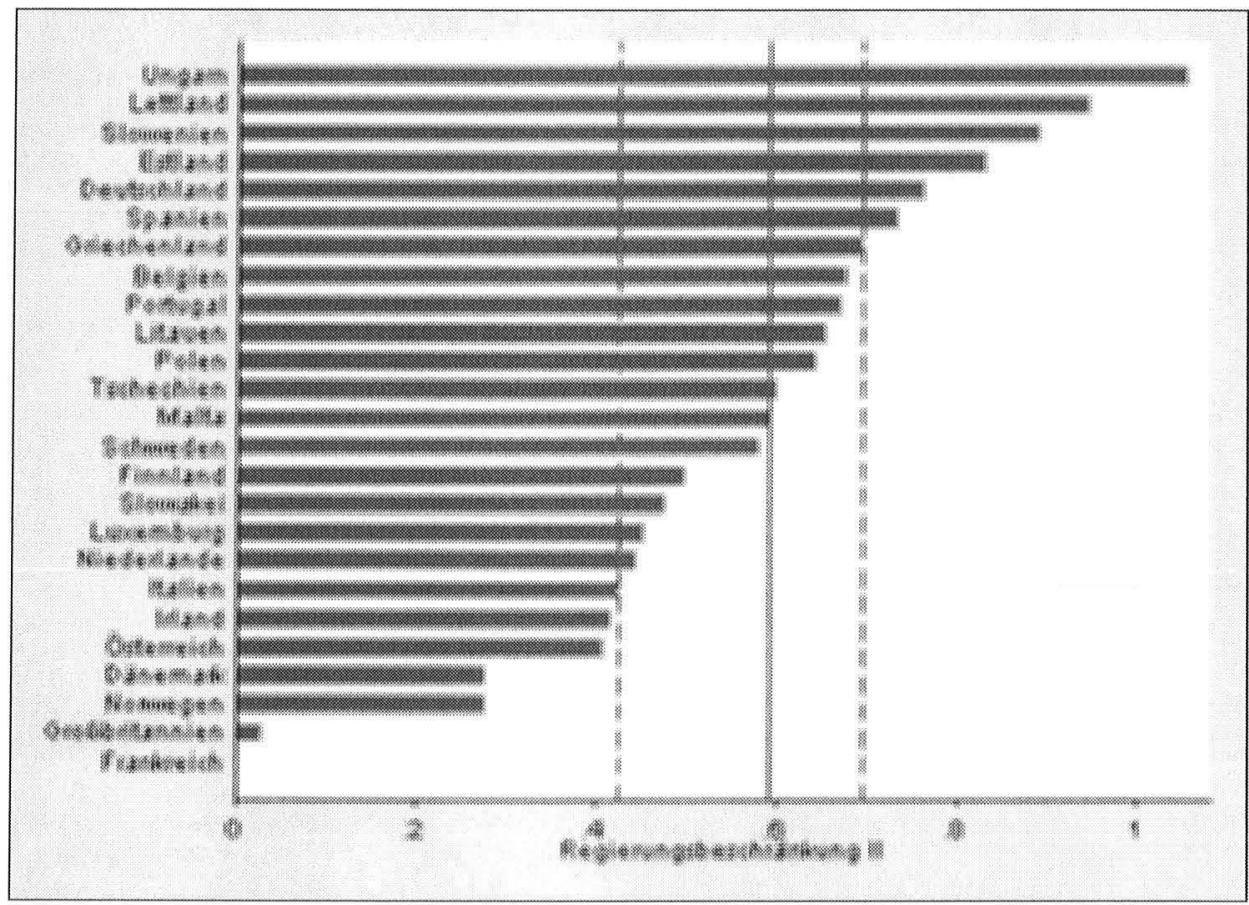

Anmerkungen: durchgezogene Linie: Medianwert, durchbrochene Linie: oberes und unteres Quartil Quelle: eigene Daten.

Diesem Index zufolge sind die Parlamente Ungarns, Lettlands und Sloweniens die stärksten Wahlorgane, während die Parlamente in Frankreich, im Vereinigten Königreich, Norwegen und Dänemark institutionell kaum in der Lage sind, ihre Regierungen durch Wahlen zu beschränken. Betrachtet man die Werte einzelner Länder, ergeben sich drei generelle Befunde: Erstens stimmen die Werte mancher Länder mit den Ergebnis-

26 Die Rangkorrelationskoeffizienten liegen etwas niedriger bei rho $=0,76$ (Wahlfreiheit und Regierungsbeschränkung I), rho = 0,71 (Wahlfreiheit und Regierungsbeschränkung II) und rho $=0,93$ (Regierungsbeschränkung I und II). Alle Zusammenhänge sind signifikant auf dem 1-Prozent-Niveau $(\mathrm{N}=25)$. 
sen existierender Forschung auf der Grundlage anderer Facetten parlamentarischer Tätigkeit überein. So erweist sich der Deutsche Bundestag als starkes Parlament; das britische Unterhaus und die französische Nationalversammlung hingegen treten als schwache Parlamente in Erscheinung. Zweitens gibt es eine Reihe überraschender Befunde. So werden das norwegische und insbesondere das dänische Parlament in der Literatur häufig als starke Parlamente beschrieben; sie sind in Bezug auf Wahlbefugnisse allerdings schwach. Ähnliches gilt in abgeschwächtem Maße für das Parlament Italiens und das der Niederlande. ${ }^{27}$ Umgekehrt weisen die Parlamente Spaniens, Griechenlands und Portugals weitreichende Wahlbefugnisse auf, was der Wahrnehmung dieser Parlamente als relativ schwach und der Systeme insgesamt als Mehrheitsdemokratien mit dominanten Regierungen widerspricht. Drittens fällt die allgemein sehr starke Stellung der osteuropäischen Parlamente auf. Sie belegen die ersten vier Plätze der Liste, und nur das slowakische Parlament liegt unterhalb des Medians. Dieses Ergebnis entspricht der Einschätzung von Länderexperten, die die Parlamente der ehemals kommunistischen EU-Staaten als sehr stark bewerten, insbesondere im Kontext der Transformation (Ágh 1997; Olson/Norton 2007).

Eine ausführliche Erklärung der starken Stellung der osteuropäischen Parlamente müsste detailliert die Entstehungsgeschichte der entsprechenden institutionellen Regelungen untersuchen und im Sinne einer Institutional-design-Logik analysieren, welche Ziele die beteiligten Akteure mit der Schaffung starker parlamentarischer Wahlbefugnisse verfolgten. Dies kann hier nicht geschehen, dennoch sollen drei mögliche Erklärungen angerissen werden, die auch einige der überraschenden Befunde für Westeuropa zumindest ansatzweise erklären könnten.

Erstens kam den Parlamenten im Transformationsprozess eine besondere Legitimität zu. Sie bildeten als charakteristischer Ausdruck pluralistischer Demokratie einen normativ hervorgehobenen Gegenpol zur gerade überwundenen Einparteidiktatur. Die Wahl externer Amtsträger durch das Parlament kann daher als Legitimierungsmechanismus für diese Ämter verstanden werden. Zweitens herrschte nach 1990 eine skeptische Grundhaltung gegenüber einer starken Exekutive. Die explizite Wahl des Kabinetts und anderer Amtsträger durch das Parlament stellt demnach eine Vorsichtsmaßnahme gegen eine erneute Monopolisierung der Macht in den Händen Weniger dar. Drittens waren die politischen Kräfteverhältnisse in und kurz nach der Umbruchphase instabil und für die Zukunft schwer vorhersehbar. In einer solchen Situation haben risikoaverse Akteure Anreize, sich einen gewissen dauerhaften Einfluss unabhängig von der künftigen Regierungsbeteiligung zu sichern (Grzymala-Busse 2006). Dies geschieht durch die Schaffung weiterer Vetopunkte und/oder Kontrollorgane, auf deren Besetzung durch Wahlen im Parlament auch Oppositionsfraktionen und Minderheiten innerhalb der Regierungsfraktionen Einfluss nehmen können. Dieser Argumentation zufolge sollten starke parlamentarische Wahlbefugnisse typischerweise nach Diktaturen und in Situationen entste-

27 Italien stellt einen Sonderfall dar, da die italienische Regierung sowohl der Deputiertenkammer als auch dem Senat verantwortlich ist. Aus Konsistenzgründen wurde auch im italienischen Fall nur die erste Kammer (= Deputiertenkammer) analysiert. Bezieht man den Senat in die Analyse mit ein, erreicht Italien etwas höhere Indexwerte und reiht sich mit einem Wert von 0,54 auf dem Index Regierungsbeschränkung II zwischen Finnland und Schweden ein. 
hen, in denen zukünftige Mehrheitsverhältnisse schwer vorhersehbar sind. Diese Erklärung passt auch zu den - angesichts des ansonsten stark mehrheitsdemokratischen Charakters dieser Länder - überraschend starken parlamentarischen Wahlbefugnissen in Spanien, Griechenland und Portugal.

Die in diesem Abschnitt festgestellten Abweichungen zwischen parlamentarischen Wahlbefugnissen und der generellen Einschätzung der Stärke einiger Parlamente zeigen bereits, dass sich die Bedeutung von Parlamenten im Hinblick auf unterschiedliche Facetten parlamentarischer Tätigkeit unterscheiden kann. Die Analyse im folgenden Abschnitt demonstriert systematisch, dass parlamentarische Wahlbefugnisse nicht nur theoretisch, sondern auch empirisch eine eigenständige Dimension institutioneller parlamentarischer Machtressourcen darstellen.

\section{Wahlbefugnisse als eigenständige Dimension parlamentarischer Macht}

Bisher wurde gezeigt, dass Wahlbefugnisse eine theoretisch relevante Dimension parlamentarischer Macht darstellen und dass sich europäische Parlamente in ihren Wahlbefugnissen signifikant unterscheiden. Dies steht im Einklang mit der seit den 1970er Jahren verbreiteten Charakterisierung von Parlamenten als multifunktionalen Institutionen (Loewenberg/Patterson 1979; Packenham 1990 [1970]). Die Unterscheidung verschiedener Parlamentsfunktionen oder (in weniger funktionalistischer Terminologie) unterschiedlicher Facetten parlamentarischer Tätigkeit sagt allerdings noch nichts darüber aus, ob diese Facetten auch empirisch distinkte Dimensionen parlamentarischer Machtbefugnisse darstellen oder ob Parlamente in unterschiedlichen Tätigkeitsfeldern konsistent stark oder schwach sind. ${ }^{28}$

Aus theoretischer Sicht spricht einiges dafür, dass institutionelle Parlamentsmacht ein auch empirisch mehrdimensionales Phänomen ist. Institutionelle Machtbefugnisse in Bezug auf die direkte Beeinflussung von Politikinhalten, die Regierungskontrolle und die Auswahl von Amtsträgern können als teilweise funktional äquivalent angesehen werden. Das fundamentale Ziel, Delegationsverluste zwischen Parlamentsmehrheit und Regierung zu minimieren, kann auf unterschiedlichen Wegen erreicht werden. Denkbar sind eine detaillierte Einflussnahme auf Gesetzesinhalte im parlamentarischen Prozess, weitreichende Delegation an die Regierung, gepaart mit einem Schwerpunkt auf die Ex-post-Kontrolle von Regierungshandeln, sowie weitreichende Delegation mit einem Fokus auf die Ex-ante-Beeinflussung von Agentenpräferenzen. Diese Wege schließen sich nicht gegenseitig aus, wohl aber ist zu erwarten, dass ihre relative Bedeutung zwischen Parlamenten variiert. Diese Vermutung führt zu der Hypothese, dass institutionelle Machtbefugnisse von Parlamenten zumindest drei distinkte Dimensionen (direkter Einfluss auf Politikinhalte, Regierungskontrolle, Wahlbefugnisse) bilden.

Diese Hypothese wird mithilfe einer konfirmatorischen Faktorenanalyse systematisch überprüft. Die beschränkte Datenlage hinsichtlich quantitativer Maße institutioneller

28 In der funktionalistischen Literatur finden sich hierzu keine Hinweise, da Unterschiede zwischen Parlamenten im Gegensatz zu generellen Mustern des Funktionswandels von Parlamenten nicht untersucht wurden. 
Machtressourcen von Parlamenten führt allerdings zu zwei Einschränkungen: Erstens können nur die westeuropäischen EU-Staaten analysiert werden. Zweitens müssen, wie in der vergleichenden Parlamentsforschung häufig der Fall, teilweise Variablen verwendet werden, die ihrerseits bereits eine Aggregation von Einzelvariablen darstellen oder aufgrund weniger Ausprägungen für faktoranalytische Verfahren nicht ideal sind (Kim/ Mueller 1994: 142-143).

Institutionelle Machtbefugnisse des Parlaments zur Beeinflussung von Politikinhalten werden über Variablen zur Agendamacht der Regierung, zum Einfluss des Parlaments im Budgetprozess und zur Stärke von Parlamentsausschüssen erfasst. Ich verwende Variablen zur Kontrolle der Tagesordnung im Plenum und den Ausschüssen sowie Instrumente zur Beendigung von Debatten (alle aus Döring 1995b), Rechte von Parlamentsausschüssen bei der Änderung von Gesetzentwürfen und deren Gesetzesinitiativrecht (beide aus Mattson/Strøm 1995) sowie einen Index zum Einfluss des Parlaments im Budgetprozess (Wehner 2006). ${ }^{29}$ Parlamentarische Machtressourcen zur Kontrolle der Regierung werden durch drei Indizes zu parlamentarischen Kontrollstrukturen, Kontrollressourcen und Kontrollrechten (alle aus Schnapp/Harfst 2005) sowie durch die Rechte von Parlamentsausschüssen zur Vorladung von Zeugen und zum Einfordern von Dokumenten (beide aus Mattson/Strøm 1995) gemessen. Schließlich gehen die drei oben entwickelten Indizes zu parlamentarischen Wahlbefugnissen in die Faktorenanalyse ein. Eine Übersicht über die Operationalisierung und den Wertebereich aller Variablen findet sich im Anhang.

Die Faktoranalyse wurde nach der Hauptachsenmethode mit anschließender Varimax-Rotation durchgeführt. Sowohl das Kaiser-Guttman-Kriterium (Eigenwert > 1) als auch die grafische Analyse des Verlaufs der Eigenwerte (scree test) führen zur Extraktion von vier Faktoren. ${ }^{30}$ Die Faktorladungen der Variablen, die Eigenwerte der einzelnen Faktoren sowie der durch sie erklärte Anteil der Gesamtvarianz sind in Tabelle 3 dargestellt.

Fast alle verwendeten Variablen laden ganz überwiegend auf nur einen Faktor, d. h. die Faktoren stehen für klar distinkte Dimensionen parlamentarischer Machtressourcen. ${ }^{31}$ Die vier extrahierten Faktoren lassen sich inhaltlich sehr gut den oben unterschie-

29 Die weiteren Variablen aus Döring (1995b) können wegen Kollinearitätsproblemen für die Faktorenanalyse nicht verwendet werden. Der bekannte Index zur Messung von Agendasetzungsmacht von Tsebelis (Tsebelis 2002: 99-105) beruht auf den auch hier verwendeten Daten von Döring und wurde daher nicht einbezogen. Alle Variablen wurden so umcodiert, dass hohe Werte für eine starke Stellung des Parlaments stehen.

30 Angesichts der geringen Fallzahl wurde die Robustheit der Eigenwerte der Faktoren zusätzlich mithilfe einer Bootstrap-Simulation überprüft (Shikano 2006). Für 2000 Bootstrap-Stichproben wurden jeweils die Eigenwerte der Faktoren und auf dieser Basis ihre Konfidenzintervalle berechnet. Da die Simulation eine deutliche Verzerrung des geschätzten Eigenwerts zeigt, wurde die Bias-corrected-and-accelerated-Methode verwendet. Die untere Grenze des so geschätzten 95-Prozent-Konfidenzintervalls liegt für die Eigenwerte aller extrahierten Faktoren über 1, d.h. die Hypothese, dass der Eigenwert in Wirklichkeit unter diesem kritischen Wert liege, kann statistisch zurückgewiesen werden.

31 Die einzigen Ausnahmen sind zwei der drei Indizes von Schnapp und Harfst, die ihrerseits auf mehreren Variablen beruhen und dabei offenbar Elemente verschiedener Dimensionen parlamentarischer Macht enthalten. 
Tabelle 3: Die Dimensionalität parlamentarischer Machtressourcen in Westeuropa

\begin{tabular}{|c|c|c|c|c|c|}
\hline & $\begin{array}{c}\text { Faktor I } \\
\text { (Politikbe- } \\
\text { einflussung) }\end{array}$ & $\begin{array}{c}\text { Faktor II } \\
\text { (Kontrolle) }\end{array}$ & $\begin{array}{l}\text { Faktor III } \\
\text { (Wahl- } \\
\text { befugnisse) }\end{array}$ & $\begin{array}{l}\text { Faktor IV } \\
\text { (Ausschuss- } \\
\text { macht) }\end{array}$ & Uniqueness \\
\hline $\begin{array}{l}\text { Parlamentarische } \\
\text { Wahlfreiheit }\end{array}$ & 0,304 & $-0,054$ & $-0,859$ & 0,028 & 0,166 \\
\hline Regierungsbeschränkung I & 0,023 & $-0,032$ & $-0,978$ & 0,057 & 0,038 \\
\hline Regierungsbeschränkung II & 0,142 & $-0,057$ & $-0,939$ & 0,200 & 0,055 \\
\hline $\begin{array}{l}\text { Kontrolle Plenaragenda } \\
\text { (Döring 1995b) }\end{array}$ & 0,888 & $-0,080$ & $-0,091$ & 0,167 & 0,169 \\
\hline $\begin{array}{l}\text { Kontrolle Ausschussagenda } \\
\text { (Döring 1995b) }\end{array}$ & 0,814 & $-0,023$ & $-0,105$ & $-0,201$ & 0,286 \\
\hline $\begin{array}{l}\text { Beendigung von } \\
\text { Plenardebatten } \\
\text { (Döring 1995b) }\end{array}$ & 0,855 & $-0,047$ & $-0,212$ & 0,263 & 0,152 \\
\hline $\begin{array}{l}\text { Einfluss auf } \\
\text { Haushaltsprozess } \\
\text { (Wehner 2006) }\end{array}$ & 0,872 & $-0,290$ & $-0,130$ & 0,130 & 0,121 \\
\hline $\begin{array}{l}\text { Kontrollstrukturen } \\
\text { (Schnapp/Harfst 2005) }\end{array}$ & 0,532 & $-0,667$ & $-0,336$ & 0,080 & 0,153 \\
\hline $\begin{array}{l}\text { Kontrollressourcen } \\
\text { (Schnapp/Harfst 2005) }\end{array}$ & $-0,125$ & 0,578 & 0,099 & 0,463 & 0,426 \\
\hline $\begin{array}{l}\text { Kontrollrechte } \\
\text { (Schnapp/Harfst 2005) }\end{array}$ & 0,107 & $-0,864$ & $-0,115$ & $-0,029$ & 0,229 \\
\hline $\begin{array}{l}\text { Vorladen von Zeugen } \\
\text { (Mattson/Strøm 1995) }\end{array}$ & 0,066 & $-0,931$ & 0,099 & 0,114 & 0,106 \\
\hline $\begin{array}{l}\text { Ausschussrechte } \\
\text { zur Änderung von } \\
\text { Gesetzentwürfen } \\
\text { (Döring 1995) }\end{array}$ & 0,249 & 0,023 & $-0,475$ & 0,765 & 0,126 \\
\hline $\begin{array}{l}\text { Gesetzesinitiativrecht } \\
\text { von Ausschüssen } \\
\text { (Mattson/Strøm 1995) }\end{array}$ & 0,207 & $-0,067$ & $-0,356$ & 0,695 & 0,342 \\
\hline $\begin{array}{l}\text { Einfordern von } \\
\text { Dokumenten } \\
\text { (Mattson/Strøm 1995) }\end{array}$ & 0,073 & $-0,131$ & 0,117 & 0,734 & 0,425 \\
\hline Eigenwert des Faktors & 5,374 & 2,516 & 1,819 & 1,497 & \\
\hline $\begin{array}{l}\text { Anteil erklärter Varianz } \\
\text { des Faktors }\end{array}$ & 0,396 & 0,185 & 0,134 & 0,110 & \\
\hline \multicolumn{6}{|c|}{$\begin{array}{l}\text { Anmerkungen: Extraktion nach der Hauptachsenmethode mit anschließender Varimax-Rotation; Zahl } \\
\text { der Faktoren bestimmt nach Kriterium Eigenwert }>1 \text { (nach Bootstrap-Simulation signifikant über 1); } \\
\text { siehe genauere Erklärung im Text. Die Zellen enthalten Faktorladungen, der betragsmäßig höchste } \\
\text { Wert jeder Variable ist fett hervorgehoben. } \mathrm{N}=15 \text { (Norwegen und die Staten der EU-15 ohne } \\
\text { Luxemburg). }\end{array}$} \\
\hline
\end{tabular}


denen Facetten parlamentarischer Tätigkeit zuordnen. Der erste Faktor erfasst institutionelle Möglichkeiten des Parlamentsplenums, Politikinhalte $\mathrm{zu}$ beeinflussen. Der zweite Faktor erfasst parlamentarische Kontrollmacht gegenüber der Regierung. ${ }^{32}$ Der dritte Faktor umfasst parlamentarische Wahlbefugnisse. Der vierte Faktor schließlich steht für Machtressourcen von Parlamentsausschüssen zur Politikbeeinflussung und Kontrolle.

Aus der Faktorenanalyse wird ersichtlich, dass institutionelle Machtbefugnisse in westeuropäischen Parlamenten tatsächlich ein mehrdimensionales Phänomen sind. Die hier erstmals systematisch untersuchten Wahlbefugnisse stellen eine dieser distinkten Dimensionen dar. Daneben werden Rechte zur Beeinflussung von Politikinhalten im Plenum sowie zur Kontrolle der Regierung als weitere Dimensionen sichtbar. Der vierte Faktor lässt sich hingegen keiner Facette parlamentarischer Tätigkeit zuordnen, sondern bezieht sich auf eine spezifische Organisationseinheit innerhalb des Parlaments. ${ }^{33}$

Aufgrund fehlender Daten konnten die untersuchten osteuropäischen Systeme nicht in die Faktorenanalyse eingeschlossen werden. Die qualitative Literatur zu parlamentarischen Machtressourcen in diesen Staaten deutet darauf hin, dass auch dort Wahlbefugnisse eine eigenständige Dimension darstellen. Dies zeigt sich, wenn man zwei in Bezug auf ihre Wahlbefugnisse besonders starke osteuropäische Parlamente, die Ungarns und Lettlands, mit zwei in dieser Hinsicht relativ schwachen Parlamenten, denen Litauens und der Tschechischen Republik, vergleicht. ${ }^{34}$ So galt das ungarische Parlament zwar im Transformationskontext als besonders stark bei der Beeinflussung von Politikinhalten (Ágh 1997); neuere Entwicklungen zeigen allerdings eine relativ schwache Stellung in Bezug auf Agendakontrollrechte und eine zunehmende Dominanz der Regierung (Ilonszki 2007). Das Ausschusssystem gilt hingegen als stark, sowohl im Hinblick auf formale Rechte als auch im Hinblick auf den Grad seiner Institutionalisierung, was größeren Einfluss auf Politikinhalte möglich erscheinen lässt (Crowther/Olson 2002; Ilonszki 2007: 51-52). Das lettische Parlament hingegen wird im Hinblick auf Agendakontrollrechte und das Ausschusssystem als relativ schwach beschrieben. Eine vergleichende Analyse der baltischen Staaten bezeichnet es explizit als schwächer als das litauische Parlament (Pettai/Madise 2006), was den Ergebnissen meiner Analyse der Wahlbefugnisse widerspricht. Das tschechische Parlament schließlich verfügt über relativ große Agendakontrollrechte und ein starkes Ausschusssystem (Crowther/Olson 2002;

32 Das Vorzeichen des Index Kontrollressourcen unterscheidet sich von den anderen Variablen des Faktors und entspricht damit nicht den theoretischen Erwartungen. Allerdings ist auch der Uniqueness-Wert dieser Variable sehr hoch; sie passt sich also insgesamt nicht gut in die durch Faktorenanalyse gewonnenen Dimensionen ein. Dies mag an der Zusammensetzung des Index liegen, der vor allem die personelle und strukturelle Ausstattung von Parlamentariern durch Mitarbeiter und Bibliotheksressourcen misst (vgl. Anhang). Diese sind zwar sicherlich wichtig zur Kontrolle von Regierungen, können aber nicht allein auf diesen Zweck reduziert werden und scheinen in keinem starken Zusammenhang mit institutionellen Kontrollrechten des Parlaments zu stehen. Dasselbe gilt für die Parlamentsgröße, die ebenfalls in den Index eingeht.

33 Bei der Interpretation dieses Faktors ist Vorsicht geboten, da die zugrunde liegenden Variablen nur über wenige Ausprägungen verfügen und zumindest die Variable zum Einfordern von Dokumenten einen hohen Uniqueness-Wert aufweist.

$34 \mathrm{Zu}$ dem noch schwächeren slowakischen Parlament liegen kaum qualitative Daten vor. 
Linek/Mansfeldová 2007) und ähnelt damit eher dem ungarischen als den beiden baltischen Parlamenten. Insgesamt scheint sich der Befund der Faktorenanalyse für die untersuchten osteuropäischen Parlamente zu bestätigen: Eine starke Stellung des Parlaments in Bezug auf Wahlbefugnisse sagt nichts über seine Stärke in anderen Tätigkeitsbereichen aus - institutionelle Parlamentsmacht ist also wohl auch in Osteuropa ein mehrdimensionales Phänomen.

Dieser Befund zeigt, dass die Beziehung zwischen Parlamenten und den von ihnen getragenen Regierungen auch in parlamentarischen Regierungssystemen unterschiedlich strukturiert sein kann. Parlamente können auf verschiedenen Wegen im politischen Alltag, d.h. diesseits der ultimativen (Drohung einer) Abberufung der Regierung, Einfluss auf Regierungshandeln und letztlich auf die Ergebnisse des politischen Entscheidungsprozesses nehmen. Als funktionale Äquivalente können sich diese Mechanismen zumindest teilweise gegenseitig substituieren. Die Parlamentsforschung hat bisher insbesondere institutionelle Möglichkeiten von parlamentarischen Akteuren zur Beeinflussung individueller Gesetze sowie in geringerem Ausmaß zur Ex-post-Kontrolle der Regierung untersucht. Dieser Beitrag zeigt, dass daneben die Auswahl von Entscheidungsträgern und Kontrolleuren einen zentralen, bisher vernachlässigten Ex-ante-Mechanismus zur Reduzierung von Delegationsverlusten darstellt.

\section{Zusammenfassung und Ausblick}

Ausgehend von der Konzeptualisierung von Parlamenten als Machtverteilungsorganen wurde in diesem Beitrag erstmals eine systematisch vergleichende Untersuchung parlamentarischer Wahlbefugnisse unternommen. Dabei wurde gezeigt, dass diese ein theoretisch wie empirisch relevantes Phänomen und eine eigenständige Dimension parlamentarischer Machtressourcen darstellen.

Die Wahl der Regierung und anderer externer Amtsträger bietet parlamentarischen Akteuren ein Instrument zur Vermeidung oder zumindest Reduzierung von Delegationsverlusten. Die Analyse von Wahlen externer Amtsträger erlaubt deren systematische Anbindung an die parlamentarische Delegationskette. Sie ermöglicht Rückschlüsse auf die Präferenzen dieser Amtsträger und damit letztlich auf die Wahrscheinlichkeit, dass diese die Regierung tatsächlich in ihrer Handlungsfreiheit einschränken. Der Grad, zu dem parlamentarische Wahlbefugnisse zur Einbeziehung weiterer Präferenzen über die der Regierung hinaus führen, lässt sich über vier institutionelle Variablen des Wahlverfahrens erfassen. Die daraus entwickelten Indizes haben sich empirisch zur Messung der institutionellen Wahlbefugnisse von 25 europäischen Parlamenten bewährt und als robust gegenüber verschiedenen Gewichtungsentscheidungen erwiesen. Mit ihrer Hilfe wurden erstmals parlamentarische Wahlbefugnisse systematisch vergleichend analysiert. Dabei wird deutlich, dass diese Befugnisse stark variieren und eine empirisch eigenständige Dimension parlamentarischer Machtressourcen bilden.

Dieser Beitrag eröffnet weitere Perspektiven für die Analyse parlamentarischer Wahlbefugnisse und ihrer Folgen. Nachdem parlamentarische Wahlbefugnisse hier über Ämter hinweg aggregiert wurden, scheint erstens eine Analyse getrennt nach Ämtern interessant, um herauszufinden, ob sich die Rolle von Parlamenten systematisch nach 
Ämtern, etwa nach deren politischer Wichtigkeit oder ihrer intendierten Überparteilichkeit, unterscheidet. Zweitens lag der Schwerpunkt in diesem Beitrag auf der Ex-anteBeeinflussung der Präferenzen externer Amtsträger. Diese Präferenzen sind aus neoinstitutionalistischer Sicht nur ein Faktor bei der Erklärung von Agentenverhalten. Um ein umfassenderes Bild des Beschränkungspotenzials dieser Amtsträger gegenüber der Regierung zu erhalten, müssten das Bestellungsverfahren, die Möglichkeiten zur Ex-postKontrolle von Amtsträgern sowie deren institutionelle Machtbefugnisse kombiniert analysiert werden. Drittens schließlich können konkrete Wahlen in Parlamenten empirisch hinsichtlich der Charakteristika gewählter Amtsträger, des Konsensgrads der Wahl und des Wahlverhaltens einzelner Akteure untersucht werden.

Aus theoretischer Sicht schließlich bietet der hier für Parlamente vorgestellte Ansatz Perspektiven für verschiedenste, durch arbeitsteilige Delegationsbeziehungen gekennzeichnete Kontexte. Dies umfasst so unterschiedliche Phänomene wie öffentliche Verwaltungen, unabhängige Regulierungsbehörden, politische Parteien, Interessenverbände und die Europäische Kommission. In all diesen Delegationsbeziehungen ist der Bestellungsmodus von Amtsträgern zentral für die Frage nach deren Präferenzen und damit letztlich auch für deren Verhalten. Bislang konzentriert sich die delegationstheoretische Forschung häufig auf Ex-post-Kontrolle durch verschiedenste Überwachungsmechanismen (monitoring) des Prinzipals und institutionelle Beschränkungen der Handlungsfreiheit bestellter Agenten. Dem Argument dieses Beitrags zufolge sollten daneben verstärkt Bestellungsverfahren und damit die Möglichkeiten der Ex-ante-Beeinflussung der Präferenzen von Amtsträgern durch den Prinzipal in den Blick genommen werden. Dies führte im Fall von Parlamenten zu neuen Einsichten bezüglich der Machtpotenziale von Prinzipalen und könnte auch über die Parlamentsforschung hinaus einen interessanten Ansatzpunkt für die theoretische wie empirische Untersuchung der Machtverteilung in Delegationsbeziehungen bieten. 
Anhang: Operationalisierungen der in der Faktoranalyse (Tabelle 3) verwendeten Variablen

\begin{tabular}{|c|c|c|}
\hline $\begin{array}{l}\text { Variable mit } \\
\text { Quelle }\end{array}$ & Operationalisierung & $\begin{array}{l}\text { Wertebereich und Ausprägung } \\
\text { der Extremwerte } \\
\text { (bei kategorialen Variablen) }\end{array}$ \\
\hline $\begin{array}{l}\text { Parlamentarische } \\
\text { Wahlfreiheit }\end{array}$ & vgl. Abschnitt 3 dieses Beitrags & $\begin{array}{l}\text { Theoretischer Wertebereich } 0-1 \\
\text { Empirischer Wertebereich } 0-0,64\end{array}$ \\
\hline $\begin{array}{l}\text { Regierungsbe- } \\
\text { schränkung I }\end{array}$ & vgl. Abschnitt 3 dieses Beitrags & $\begin{array}{l}\text { Theoretischer Wertebereich } 0-2 \\
\text { Empirischer Wertebereich } 0-87\end{array}$ \\
\hline $\begin{array}{l}\text { Regierungsbe- } \\
\text { schränkung II }\end{array}$ & vgl. Abschnitt 3 dieses Beitrags & $\begin{array}{l}\text { Theoretischer Wertebereich } \\
0-2,33 \\
\text { Empirischer Wertebereich } 0-1,06\end{array}$ \\
\hline $\begin{array}{l}\text { Kontrolle der } \\
\text { Plenaragenda } \\
\text { (Döring 1995) }\end{array}$ & $\begin{array}{l}\text { Rechte der Regierung zur Festlegung der } \\
\text { Tagesordnung im Plenum }\end{array}$ & $\begin{array}{l}\text { Wertebereich } 1-7 \\
1=\text { Regierung allein bestimmt } \\
\text { Agenda } \\
7=\text { Parlament allein bestimmt } \\
\text { Agenda }\end{array}$ \\
\hline $\begin{array}{l}\text { Kontrolle der } \\
\text { Ausschussagenda } \\
\text { (Döring 1995) }\end{array}$ & $\begin{array}{l}\text { Rechte der Ausschüsse zur Kontrolle ihrer } \\
\text { Tagesordnung }\end{array}$ & $\begin{array}{l}\text { Wertebereich } 1-4 \\
1=\text { vom Plenum an den Aus- } \\
\text { schuss überwiesene Vorlagen } \\
\text { stellen automatisch die Tages- } \\
\text { ordnung des Ausschusses dar } \\
4=\text { Plenum kann dem Ausschuss } \\
\text { Gesetzentwürfe nicht wieder } \\
\text { entziehen }\end{array}$ \\
\hline $\begin{array}{l}\text { Beendigung von } \\
\text { Plenardebatten } \\
\text { (Döring 1995) }\end{array}$ & $\begin{array}{l}\text { Regeln zur Beendigung der Plenardebatte vor } \\
\text { der Abstimmung }\end{array}$ & $\begin{array}{l}\text { Wertebereich } 1-3 \\
1=\text { Debattenlänge kann per } \\
\text { Mehrheitsentscheidung festgelegt } \\
\text { werden } \\
3=\text { keine vorzeitige Beendigung } \\
\text { möglich }\end{array}$ \\
\hline $\begin{array}{l}\text { Einfluss auf } \\
\text { Haushaltsprozess } \\
\text { (Wehner 2006) }\end{array}$ & $\begin{array}{l}\text { Index auf der Grundlage von Daten der } \\
\text { „Survey on Budget Practices and Procedures“ } \\
\text { von OECD und Weltbank (2003). Der Index } \\
\text { errechnet sich aus sechs Variablen zu (1) } \\
\text { Rechten des Parlaments zur Änderung des } \\
\text { Haushaltsentwurfs, (2) Rückfallregelungen für } \\
\text { den Fall gescheiterter Haushaltsverhandlun- } \\
\text { gen, (3) der Flexibilität der Regierung bei der } \\
\text { Umsetzung des Haushaltsgesetzes, (4) dem } \\
\text { Zeitraum, der dem Parlament zur Prüfung und } \\
\text { Bearbeitung des Haushaltsentwurfs zur Ver- } \\
\text { fügung steht, (5) den mit dem Haushaltspro- } \\
\text { zess befassten Parlamentsausschüssen sowie } \\
\text { (6) der Existenz parlamentarischer Forschungs- } \\
\text { dienste zu Haushaltsfragen. Die ersten drei } \\
\text { und letzten drei Variablen werden additiv } \\
\text { aggregiert, die entstehenden Teilindizes multi- } \\
\text { plikativ verknüpft. }\end{array}$ & $\begin{array}{l}\text { Theoretischer Wertebereich } \\
0-100 \\
\text { Empirischer Wertebereich } \\
16,7-88,9 \text { für die von Wehner } \\
\text { untersuchten Fälle } \\
\text { Empirischer Wertebereich } \\
\text { 16,7-65,3 für die hier analysier- } \\
\text { ten Fälle }\end{array}$ \\
\hline
\end{tabular}




\begin{tabular}{|c|c|c|}
\hline $\begin{array}{l}\text { Variable mit } \\
\text { Quelle }\end{array}$ & Operationalisierung & $\begin{array}{l}\text { Wertebereich und Ausprägung } \\
\text { der Extremwerte } \\
\text { (bei kategorialen Variablen) }\end{array}$ \\
\hline $\begin{array}{l}\text { Kontrollstrukturen } \\
\text { (Schnapp/Harfst } \\
\text { 2005) }\end{array}$ & $\begin{array}{l}\text { Index auf der Grundlage von Daten zu (1) } \\
\text { Anzahl der Ausschüsse, (2) Größe der Aus- } \\
\text { schüsse, (3) Spiegelbildlichkeit von Ausschüs- } \\
\text { sen und Ministerien sowie (4) Anzahl der } \\
\text { Ausschussmitgliedschaften pro Abgeordnetem. } \\
\text { Der Index wird konstruiert als Mittelwert der } \\
\text { Rangplätze der Parlamente auf den jeweiligen } \\
\text { Einzelindikatoren. } \\
\text { Das Vorzeichen der Indexwerte wurde vom } \\
\text { Autor durch Multiplikation mit dem Faktor } \\
\text {-1 umgekehrt, damit hohe Werte wie bei den } \\
\text { übrigen Variablen für starke Parlamente stehen. }\end{array}$ & $\begin{array}{l}\text { Theoretischer Wertebereich } 1-21 \\
\text { Empirischer Wertebereich } \\
3,3-13,9\end{array}$ \\
\hline $\begin{array}{l}\text { Kontroll- } \\
\text { ressourcen } \\
\text { (Schnapp/Harfst } \\
\text { 2005) }\end{array}$ & $\begin{array}{l}\text { Index auf der Grundlage von Daten zu (1) Par- } \\
\text { lamentsgröße, (2) Größe der Mitarbeiterstäbe } \\
\text { des Parlaments, (3) personeller Unterstützung } \\
\text { der Ausschüsse sowie (4) Bibliotheken und } \\
\text { wissenschaftlichen Diensten der Parlamente. } \\
\text { Der Index wird konstruiert als Mittelwert der } \\
\text { Rangplätze der Parlamente auf den jeweiligen } \\
\text { Einzelindikatoren. } \\
\text { Das Vorzeichen der Indexwerte wurde vom } \\
\text { Autor durch Multiplikation mit dem Faktor -1 } \\
\text { umgekehrt, damit hohe Werte wie bei den übri- } \\
\text { gen Variablen für starke Parlamente stehen. }\end{array}$ & $\begin{array}{l}\text { Theoretischer Wertebereich 1-21 } \\
\text { Empirischer Wertebereich } \\
\text { 2,4-18,4 für die von Schnapp/ } \\
\text { Harfst untersuchten Fälle } \\
\text { Empirischer Wertebereich } \\
5,3-16,0 \text { für die hier analysierten } \\
\text { Fälle }\end{array}$ \\
\hline $\begin{array}{l}\text { Kontrollrechte } \\
\text { (Schnapp/Harfst } \\
\text { 2005) }\end{array}$ & $\begin{array}{l}\text { Index auf der Grundlage von Daten zu (1) } \\
\text { Informationsrechten der Ausschüsse, (2) Zeit } \\
\text { des Parlaments zur Prüfung von Regierungs- } \\
\text { vorlagen im Budgetprozess sowie (3) Existenz } \\
\text { unabhängiger Rechnungsprüfungs- und } \\
\text { Ombudsstellen. Der Index wird konstruiert } \\
\text { als Mittelwert der Rangplätze der Parlamente } \\
\text { auf den jeweiligen Einzelindikatoren. } \\
\text { Das Vorzeichen der Indexwerte wurde vom } \\
\text { Autor durch Multiplikation mit dem Faktor } \\
\text {-1 umgekehrt, damit hohe Werte wie bei den } \\
\text { übrigen Variablen für starke Parlamente stehen. }\end{array}$ & $\begin{array}{l}\text { Theoretischer Wertebereich 1-21 } \\
\text { Empirischer Wertebereich } \\
6,4-16,1 \text { für die von Schnapp/ } \\
\text { Harfst untersuchten Fälle } \\
\text { Empirischer Wertebereich } \\
7,0-16,1 \text { für die hier analysierten } \\
\text { Fälle }\end{array}$ \\
\hline $\begin{array}{l}\text { Vorladen von } \\
\text { Zeugen (Mattson/ } \\
\text { Strøm 1995) }\end{array}$ & $\begin{array}{l}\text { Recht der Parlamentsausschüsse, Zeugen vor- } \\
\text { zuladen und ihr Kommen zu erzwingen }\end{array}$ & $\begin{array}{l}\text { Dummyvariable } \\
0=\text { nicht vorhanden } \\
1=\text { vorhanden }\end{array}$ \\
\hline $\begin{array}{l}\text { Ausschussrechte } \\
\text { zur Änderung } \\
\text { von Gesetz- } \\
\text { entwürfen } \\
\text { (Döring 1995) }\end{array}$ & $\begin{array}{l}\text { Rechte der Parlamentsausschüsse, } \\
\text { Gesetzentwürfe umzuschreiben }\end{array}$ & $\begin{array}{l}\text { Wertebereich } 1-4 \\
1=\text { Plenum stimmt über } \\
\text { ursprünglichen Entwurf ab } \\
4=\text { Ausschüsse können Entwurf } \\
\text { frei verändern und das Plenum } \\
\text { stimmt über den geänderten } \\
\text { Entwurf ab }\end{array}$ \\
\hline $\begin{array}{l}\text { Gesetzesinitiativ- } \\
\text { recht von Aus- } \\
\text { schüssen (Mattson/ } \\
\text { Strøm 1995) }\end{array}$ & $\begin{array}{l}\text { Rechte der Parlamentsausschüsse, } \\
\text { Gesetzentwürfe einzubringen }\end{array}$ & $\begin{array}{l}\text { Wertebereich } 1-3 \\
1=\text { keinerlei Initiativrecht } \\
3=\text { freies Initiativrecht }\end{array}$ \\
\hline $\begin{array}{l}\text { Einfordern von } \\
\text { Dokumenten } \\
\text { (Mattson/Strøm } \\
\text { 1995) }\end{array}$ & $\begin{array}{l}\text { Recht der Parlamentsausschüsse, } \\
\text { Dokumente einzufordern }\end{array}$ & $\begin{array}{l}\text { Dummyvariable } \\
0=\text { nicht vorhanden } \\
1=\text { vorhanden }\end{array}$ \\
\hline
\end{tabular}




\section{Literatur}

Ágh, Attila, 1997: Parliaments as Policy-Making Bodies in East Central Europe. The Case of Hungary, in: International Political Science Review 18, 417-432.

Andeweg, Rudy B./Nijzink, Lia, 1995: Beyond the Two-Body Image. Relations Between Ministers and MPs, in: Döring, Herbert (Hrsg.), Parliaments and Majority Rule in Western Europe. Frankfurt: Campus Verlag, 154-178.

Andeweg, Rudy B., 2000: Ministers as Double Agents? The Delegation Process Between Cabinet and Ministers, in: European Journal of Political Research 37, 377-395.

Bagehot, Walter, 1964 [1867]: The English Constitution. London: Cambridge University Press.

Behnke, Joachim, 2005: Lassen sich Signifikanztests auf Vollerhebungen anwenden? Einige essayistische Anmerkungen, in: Politische Vierteljahresschrift 46, O-1-O-15.

Bergman, Torbjörn, 1993: Formation Rules and Minority Governments, in: European Journal of Political Research 23, 55-66.

Bergman, Torbjörn/Müller, Wolfgang C./Strøm, Kaare/Blomgren, Magnus, 2003: Democratic Delegation and Accountability. Cross-national Patterns, in: Strøm, Kaare/Müller, Wolfgang C./ Bergman, Torbjörn (Hrsg.), Delegation and Accountability in Parliamentary Democracies. Oxford: Oxford University Press, 109-220.

Broscheid, Andreas/Gschwend, Thomas, 2005: Zur statistischen Analyse von Vollerhebungen, in: Politische Vierteljahresschrift 46, O-16-O-28.

Bryce, James, 1921: Modern Democracies. New York: Macmillan.

Carroll, Royce/Cox, Gary W./Pachón, Mónica, 2006: How Parties Create Electoral Democracy, Chapter 2, in: Legislative Studies Quarterly 31, 153-174.

Chang, Kelly H., 2001: The President versus the Senate. Appointments in the American System of Separated Powers and the Federal Reserve, in: Journal of Law, Economics, and Organization 17, 319-355.

Cox, Gary W., 1987a: The Efficient Secret. The Cabinet and the Development of Political Parties in Victorian England. Cambridge: Cambridge University Press.

Cox, Gary W., 1987b: Electoral Equilibrium under Alternative Voting Institutions, in: American Journal of Political Science 31, 82-108.

Crowther, William E./Olson, David M., 2002: Committee Systems in New Democratic Parliaments. Comparative Institutionalization, in: Olson, David M./Crowther, William E. (Hrsg.), Committees in Post-Communist Democratic Parliaments. Columbus: Ohio State University Press, 171-206.

Döring, Herbert, 1994: Parlament und Regierung, in: Gabriel, Oscar W./Brettschneider, Frank (Hrsg.), Die EU-Staaten im Vergleich. Strukturen, Prozesse, Politikinhalte, 2. Auflage. Bonn: Bundeszentrale für politische Bildung, 336-358.

Döring, Herbert (Hrsg.), 1995a: Parliaments and Majority Rule in Western Europe. Frankfurt: Campus Verlag.

Döring, Herbert, 1995b: Time as a Scarce Resource. Government Control of the Agenda, in: Döring, Herbert (Hrsg.), Parliaments and Majority Rule in Western Europe. Frankfurt: Campus Verlag, 223-246.

Döring, Herbert/Hallerberg, Mark (Hrsg.), 2004: Patterns of Parliamentary Behaviour. Passage of Legislation across Western Europe. Burlington: Ashgate.

Epstein, Lee/Segal, Jeffrey A., 2005: Advice and Consent. The Politics of Judicial Appointments. Oxford: Oxford University Press.

Fearon, James D., 1999: Electoral Accountability and the Control of Politicians. Selecting Good Types versus Sanctioning Poor Performance, in: Przeworski, Adam/Stokes, Susan C./Manin, Bernard (Hrsg.), Democracy, Accountability, and Representation. Cambridge: Cambridge University Press, 55-97. 
Gallagher, Michael/Laver, Michael/Mair, Peter, 2006: Representative Government in Modern Europe, 4. Auflage. Boston: Mcgraw-Hill Higher Education.

Grzymala-Busse, Anna, 2006: The Discreet Charm of Formal Institutions. Postcommunist Competition and State Oversight, in: Comparative Political Studies 39, 271-300.

Helms, Ludger, 2005: Der Wandel politischer Kontrolle in den parlamentarischen Demokratien Westeuropas, in: Zeitschrift für Parlamentsfragen 36, 390-410.

Hinich, Melvin J./Munger, Michael C., 1997: Analytical Politics. Cambridge: Cambridge University Press.

Hönnige, Christoph, 2007: Verfassungsgericht, Regierung und Opposition. Die vergleichende Analyse eines Spannungsdreiecks. Wiesbaden: VS Verlag für Sozialwissenschaften.

Ilonszki, Gabriella, 2007: From Minimal to Subordinate. A Final Verdict? The Hungarian Parliament, 1990-2002, in: Journal of Legislative Studies 13, 38-58.

Ishiyama Smithey, Shannon/Ishiyama, John, 2002: Judicial Activism in Post-Communist Politics, in: Law \& Society Review 36, 719-741.

Ismayr, Wolfgang (Hrsg.), 1999: Die politischen Systeme Westeuropas, 2. Auflage. Opladen: Leske + Budrich

Ismayr, Wolfgang (Hrsg.), 2004: Die politischen Systeme Osteuropas, 2. Auflage. Opladen: Leske + Budrich

Jenny, Marcelo/Müller, Wolfgang C., 1995: Presidents of Parliaments. Neutral Chairmen or Assets of the Majority? in: Döring, Herbert (Hrsg.), Parliaments and Majority Rule in Western Europe. Frankfurt: Campus Verlag, 326-364.

Kaiser, André, 2007: Neuere Beiträge zur empirischen Institutionenanalyse. Von der Gegenstandszur Problemorientierung, in: Politische Vierteljahresschrift 48, 119-135.

Kim, Jae-On/Mueller, Charles W., 1994: Factor Analysis. Statistical Methods and Practical Issues, in: Lewis-Beck, Michael S. (Hrsg.), Factor Analysis and Related Techniques. London: SAGE, 75-155.

Krehbiel, Keith, 2006: Pivots, in: Weingast, Barry R./Wittman, Donald A. (Hrsg.), The Oxford Handbook of Political Economy. Oxford: Oxford Unversity Press, 223-240.

Krehbiel, Keith, 2007: Supreme Court Appointments as a Move-the-Median Game, in: American Journal of Political Science 51, 231-240.

Linek, Lukás/Mansfeldová, Zdenka, 2007: The Parliament of the Czech Republic, 1993-2004, in: Journal of Legislative Studies 13, 12-37.

Loewenberg, Gerhard/Patterson, Samuel C., 1979: Comparing Legislatures. Boston: Little Brown.

Lupia, Arthur, 2003: Delegation and its Perils, in: Strøm, Kaare/Müller, Wolfgang C./Bergman, Torbjörn (Hrsg.), Delegation and Accountability in Parliamentary Democracies. Oxford: Oxford University Press, 33-54.

Martin, Lanny W./Vanberg, Georg, 2005: Coalition Policymaking and Legislative Review, in: American Political Science Review 99, 93-106.

Mattson, Ingvar/Strøm, Kaare, 1995: Parliamentary Committees, in: Döring, Herbert (Hrsg.), Parliaments and Majority Rule in Western Europe. Frankfurt: Campus Verlag, 249-307.

Miller, Bernhard, 2004: Der Ombudsman. Ein Instrument der Verwaltungskontrolle im Vergleich von 36 Ländern. Unveröffentlichte Magisterarbeit. Mannheim.

Miller, Bernhard, 2007: Maßvoll messen. Zur konzeptionellen Entwicklung von Messinstrumenten, in: Gschwend, Thomas/Schimmelfennig, Frank (Hrsg.), Forschungsdesign in der Politikwissenschaft. Probleme - Strategien - Anwendungen. Frankfurt: Campus Verlag, 123-148

Moraski, Bryon J./Shipan, Charles R., 1999: The Politics of Supreme Court Nominations: A Theory of Institutional Constraints and Choices, in: American Journal of Political Science 43, 1069-1095.

Müller, Wolfgang C., 2000: Political Parties in Parliamentary Democracies. Making Delegation and Accountability Work, in: European Journal of Political Research 34, 309-333. 
Müller, Wolfgang C./Bergman, Torbjörn/Strøm, Kaare, 2003: Parliamentary Democracy: Promise and Problems, in: Strøm, Kaare/Müller, Wolfgang C./Bergman, Torbjörn (Hrsg.), Delegation and Accountability in Parliamentary Democracies. Oxford: Oxford University Press, 3-32.

National Audit Office, 2005: State Audit in the European Union. London: National Audit Office.

Neto, Octavio Amorim/Strøm, Kaare, 2006: Breaking the Parliamentary Chain of Delegation. Presidents and Non-partisan Cabinet Members in European Democracies, in: British Journal of Political Science 36, 619-643.

Nixon, David C., 2004: Separation of Powers and Appointee Ideology, in: Journal of Law, Economics, and Organization 20, 438-457.

Norton, Philip, 1990: Parliaments. A Framework for Analysis, in: Norton, Philip (Hrsg.), Parliaments in Western Europe. London: Frank Cass Publishers, 1-9.

Olson, David M./Norton, Philip, 2007: Post-Communist and Post-Soviet Parliaments. Divergent Paths from Transition, in: Journal of Legislative Studies 13, 164-196.

Packenham, Robert A., 1990 [1970]: Legislatures and Political Development, in: Norton, Philip (Hrsg.), Legislatures. Oxford: Oxford University Press, 81-96.

Patzelt, Werner J., 2003: Parlamente und ihre Funktionen, in: Patzelt, Werner J. (Hrsg.), Parlamente und ihre Funktionen. Institutionelle Mechanismen und institutionelles Lernen im Vergleich. Wiesbaden: Westdeutscher Verlag, 13-49.

Patzelt, Werner J., 2004: Parlamentarismus, in: Helms, Ludger/Jun, Uwe (Hrsg.), Politische Theorie und Regierungslehre. Eine Einführung in die politikwissenschaftliche Institutionenforschung. Frankfurt: Campus Verlag, 97-129.

Pettai, Vello/Madise, Ülle, 2006: The Baltic Parliaments. Legislative Performance from Independence to EU Accession, in: Journal of Legislative Studies 12, 291-310.

Przeworski, Adam/Stokes, Susan C./Manin, Bernard (Hrsg.), 1999: Democracy, Accountability, and Representation. Cambridge: Cambridge University Press.

Romer, Thomas/Rosenthal, Howard, 1978: Political Resource-Allocation, Controlled Agendas, and the Status Quo, in: Public Choice 33, 27-44.

Saalfeld, Thomas, 1995: On Dogs and Whips. Recorded Votes, in: Döring, Herbert (Hrsg.), Parliaments and Majority Rule in Western Europe. Frankfurt: Campus Verlag, 528-565.

Schnapp, Kai-Uwe/Harfst, Philipp, 2005: Parlamentarische Informations- und Kontrollressourcen in 22 westlichen Demokratien, in: Zeitschrift für Parlamentsfragen 36, 348-370.

Schulze-Fielitz, Helmuth, 1984: Der informale Verfassungsstaat. Aktuelle Beobachtungen des Verfassungslebens der Bundesrepublik Deutschland im Lichte der Verfassungstheorie. Berlin: Duncker \& Humblot.

Schüttemeyer, Suzanne S., 2003: Vergleichende Parlamentarismusforschung, in: Berg-Schlosser, Dirk/Müller-Rommel, Ferdinand (Hrsg.), Vergleichende Politikwissenschaft, 4. Auflage. Opladen: Leske + Budrich, 207-227.

Shikano, Susumu, 2006: Bootstrap und Jackknife, in: Behnke, Joachim/Gschwend, Thomas/ Schindler, Delia/Schnapp, Kai-Uwe (Hrsg.), Methoden der Politikwissenschaft. Neuere qualitative und quantitative Analyseverfahren. Baden-Baden: Nomos, 69-79.

Siaroff, Alan, 2003: Comparative Presidencies. The Inadequacy of the Presidential, SemiPresidential and Parliamentary Distinction, in: European Journal of Political Research 42, 287-312.

Sieberer, Ulrich, 2006: Party Unity in Parliamentary Democracies. A Comparative Analysis, in: Journal of Legislative Studies 12, 150-178.

Sieberer, Ulrich, 2008: Können ja - wollen nein? Die Anreize außerparlamentarischer Amtsträger zur Beschränkung der Regierung, in: Schöne, Helmar/von Blumenthal, Julia (Hrsg.), Parlamentarismusforschung in Deutschland. Ergebnisse und Perspektiven 40 Jahre nach Erscheinen von Gerhard Loewenbergs Standardwerk zum Deutschen Bundestag. Baden-Baden: Nomos. 
Steffani, Winfried, 1997: Gewaltenteilung und Parteien im Wandel. Wiesbaden: Westdeutscher Verlag.

Strøm, Kaare, 2000: Delegation and Accountability in Parliamentary Democracies, in: European Journal of Political Research 37, 261-289.

Strøm, Kaare, 2003: Parliamentary Democracy and Delegation, in: Strøm, Kaare/Müller, Wolfgang C./Bergman, Torbjörn (Hrsg.), Delegation and Accountability in Parliamentary Democracies. Oxford: Oxford University Press, 55-106.

Strøm, Kaare/Müller, Wolfgang C./Bergman, Torbjörn (Hrsg.), 2003a: Delegation and Accountability in Parliamentary Democracies. Oxford: Oxford University Press.

Strøm, Kaare/Müller, Wolfgang C./Bergman, Torbjörn/Nyblade, Benjamin, 2003b: Dimensions of Citizen Control, in: Strøm, Kaare/Müller, Wolfgang C./Bergman, Torbjörn (Hrsg.), Delegation and Accountability in Parliamentary Democracies. Oxford: Oxford University Press, 651706.

Tsebelis, George, 2002: Veto Players. How Political Institutions Work. Princeton: Princeton University Press.

von Beyme, Klaus, 1997: Der Gesetzgeber. Der Bundestag als Entscheidungszentrum. Opladen: Westdeutscher Verlag.

von Beyme, Klaus, 1999: Die Parlamentarische Demokratie. Entstehung und Funktionsweise 1989-1999, 3. Auflage. Opladen: Leske + Budrich.

Wehner, Joachim, 2006: Assessing the Power of the Purse. An Index of Legislative Budget Institutions, in: Political Studies 54, 767-785. 